\title{
TENSOR ALGEBRAS OVER HIIBERT SPACES. I
}

\author{
BY \\ I. E. SEGAL $\left({ }^{1}\right)$ \\ INTRODUCTION
}

We treat the algebra of all covariant tensors over a complex Hilbert space $\mathcal{C}$ and particularly the quotient algebras of it modulo certain completely characteristic ideals. These are ideals that are invariant both under the automorphisms of the algebra and also under the continuous linear operators that commute with the automorphisms. The only maximally nontrivial such ideals are the apparent ones, leading to the algebras of symmetric and skewsymmetric tensors.

The bulk of the present paper is devoted to an investigation of the symmetric algebra, which is shown to be unitarily equivalent to the space $L_{2}\left(\mathcal{F C}^{\prime}\right)$ of "square-integrable functionals" over a real Hilbert space $\mathfrak{H C}^{\prime}$ of which $\mathfrak{H C}$ is the complexification. This equivalence is canonical and greatly clarifies the structure of various linear operators on the algebras. Among these is the Plancherel transform, which, like some technically cogent features of the theory of Lebesgue integration, is extended from the classical case when $\mathfrak{F C}^{\prime}$ is finite-dimensional to the case of a space of arbitrary dimension.

These developments relate to such varied matters as quantum field theory, stochastic processes, and euclidean analysis. It therefore seems appropriate, for clarity, to number and collect together at the end of the paper all detailed references to the existing literature. Our own work arose as an abstraction and elaboration of quantum field statistics $\left({ }^{2}\right)$.

\section{Characteristic ideals in tensor algebras}

1. Notation and technical preliminaries. Throughout the following, $\mathfrak{H C}$ will represent a complex Hilbert space of arbitrary dimension. The space of all covariant $n$-tensors $\left.{ }^{3}\right)$ over $\mathfrak{H C}$ (i.e. the $n$-fold tensor product of $\mathfrak{H C}$ with itself) will be denoted by $\mathfrak{H}_{n}(n=1,2, \cdots)$, and $\mathfrak{K}_{1}$ will be identified with $\mathfrak{H}$. The

Presented to the Society, December 28, 1954; received by the editors April 25, 1955.

(1) The author is indebted to the Guggenheim Foundation and to the Office of Naval Research for support during the preparation of parts of this paper.

(2) Previous use of tensors over Hilbert space in connection with quantum field theory has been made by J. M. Cook [2]. This paper includes a thorough treatment of properties of the annihilation and creation operators, and of the differentials $d \Gamma$ of the canonical unitary representations (see below). However, its results are not logically necessary for the present paper.

( ${ }^{3}$ Concerning tensors over a Hilbert space, see [7]. By "covariant" tensor over a space is here meant one that transforms in the same fashion as vectors in the space, rather than contragrediently to such vectors. 
space of all complex numbers with the inner product $(a, b)=a b$ will be denoted by $\mathfrak{F}_{0}$. If $x$ and $y$ are (covariant) $r$ - and $s$-tensors respectively, their tensor product is an $(r+s)$ tensor denoted as $x \times y$, the product being defined as scalar multiplication when one of the tensors is of rank zero. Putting $K$ for the Hilbert space direct sum $\sum_{n=0}^{\infty} \mathfrak{F C}_{n}, K$ contains as a dense subset the algebraic direct sum $K_{0}$ of the $\mathfrak{H}_{n}$, consisting of the sums $\sum_{n} x_{n}$ with $x_{n} \in \mathfrak{H}_{n}$ and $x_{n}=0$ for large $n$. (When the range of a summation or integration is unspecified, it is understood to be the entire range of the relevant variable.) Tensor multiplication extends uniquely by linearity to a multiplication on $K_{0}$ that is associative, distributive, and bilinear. Multiplication may be defined more generally for suitable tensors $x$ and $y$ as follows. Denoting the component in $\mathcal{H C}_{n}$ of an arbitrary element $u$ of $K$ by $u_{n}$, let $z_{n}=\sum_{r=0}^{n} x_{r} \times y_{n-r}$. If there exists an element $z$ in $K$ with components $z_{n}$ (i.e., if $\sum_{n}\left|z_{n}\right|^{2}$ is finite), then $x \times y$ is said to be defined and to have the value $z$. By the algebra of covariant tensors over $\mathfrak{H C}$ will be meant the system constituted of the Hilbert space $K$, the gradation of $K$ as $\sum_{n} \mathcal{F C}_{n}$ (more specifically, the distinguished decomposition of $K$ as an orthogonal direct sum of subspaces indexed by the non-negative integers), and the tensor multiplication on $K$.

An automorphism of $K$ is consequently a unitary that leaves $\mathcal{H}_{n}$ invariant and preserves tensor multiplication. In particular, the contraction to $\mathfrak{T}$ of an automorphism coincides with a unitary $U$ on $\mathcal{H}$. Now $K$ is invariantly associated with $\mathfrak{H}$, so that an isomorphism $U$ of $\mathfrak{H C}$ onto $\mathfrak{H C}$ has an extension $\Gamma(U)$ to an automorphism of $K$. As it is readily verified that the only automorphism of $K$ that leaves the elements of $\mathfrak{H C}$ fixed is the identity, every automorphism of $K$ has the form $\Gamma(U)$ for some unitary $U$ on $\mathfrak{H C}$. In fact there is no difficulty in showing that the mapping $U \rightarrow \Gamma(U)$ is an isomorphism of the unitary group on $\mathcal{H C}$ onto the automorphism group of $K$, both groups bearing the strong operator topology.

The canonical representation of the symmetric group $\Sigma_{n}$ of degree $n$ on the space $\mathfrak{H C}_{n}$ of $n$-tensors over $\mathfrak{H C}$ is defined as the unitary representation $V^{(n)}$ determined uniquely by the condition that $V^{(n)}(\pi)\left(y_{1} \times y_{2} \times \cdots \times y_{n}\right)$ $=y_{\pi(1)} \times y_{\pi(2)} \times \cdots \times y_{\pi(n)}$ for arbitrary vectors $y_{1}, y_{2}, \cdots, y_{n}$ in $\mathcal{H C}$ and permutation $\pi$ in $\sum_{n}$. The operators $S_{n}=(n !)^{-1} \sum_{\pi} V^{(n)}(\pi)$ and $A_{n}=(n !)^{-1} \sum_{\pi} \operatorname{sgn}(\pi) V^{(n)}(\pi)$, which are easily seen to be projections, are called symmetrization and alternation, respectively, on $\mathcal{F C}_{n}$. The direct sums $S=\sum_{n} S_{n}$ and $A=\sum_{n} A_{n}$ (where $S_{0}$ and $A_{0}$ are both defined as the identity operator on $\mathfrak{C}_{0}$ ) are called simply symmetrization and alternation (on $K$ ). The null spaces of $S$ and of $A$ are called the symmetric and skew-symmetric ideals of $K$ (see below).

$K$ is an example of a g- (graded) algebra, where

Definition 1. A g-algebra is a system constituted of a Hilbert space $\mathcal{L}$, a gradation of $\mathcal{L}$ as a direct sum $\sum_{n=0}^{\infty} \mathcal{L}_{n}$, and a bilinear multiplication between $\mathcal{L}_{r}$ and $\mathcal{L}_{\text {s }}$ into $\mathcal{L}_{r+s}$, satisfying the conditions that multiplication is 
associative and satisfies the inequality $|x y| \leqq|x||y| ; \mathcal{L}_{0}$ is one-dimensional and contains a unit for multiplication of unit norm; $\mathcal{L}$ is generated (algebraically and topologically) by $\mathcal{L}_{0}$ and $\mathcal{L}_{1}$.

"Subspace" (of a Hilbert space) will mean closed linear submanifold. A subspace $\mathcal{X}$ of a g-algebra $\mathcal{L}$ is said to be graded in case $\mathscr{X}=\sum_{n} \mathcal{X}_{n}$, with $\mathcal{H}_{n} \subset \mathcal{L}_{n}$. A subspace is a (left/two-sided) "ideal" if it is invariant under multiplication by the elements of $\mathcal{L}_{1}$ (on the left/on both sides). It is not difficult to show that an ideal is necessarily invariant under multiplication by the elements of any $\mathcal{L}_{n}$. From this point, "ideal" will mean two-sided ideal, unless otherwise specified. Now if $\mathcal{X}$ is a graded ideal in $\mathcal{L}$, the quotient Hilbert space $\mathcal{L} / \mathscr{X}$ is graded as $\sum_{n}\left(\mathcal{L}_{n} / \mathcal{X}_{n}\right)$. Defining $(x+\mathscr{X})(y+\mathcal{X})=x y+\mathcal{X}$ for $x \in \mathcal{X}_{r}$ and $y \in \mathcal{X}_{s}$, it is readily verified that $\mathcal{L} / \mathcal{X}$ becomes a g-algebra.

"Ring" (of operators) on $\mathfrak{H C}$ will mean a weakly closed self-adjoint ring of continuous linear operators on $\mathcal{H C}$ containing the identity operator.

2. Creation operators and tensor ideals. In this section the so-called creation and annihilation operators are discussed, partly for motivation and partly for their technical relevance.

A "quantum field statistics" (of identical particles with wave functions in a Hilbert space $\mathfrak{F}$ ) has roughly two basic features:

(1) a continuous representation $\Xi$ of the unitary group on $\mathcal{F}$ by unitary operators on a Hilbert space $\mathcal{L}$ (of wave functions of the quantum field)

(2) operators $C(x)$ on $\mathcal{L}$ that represent the creation of a particle with wave function $x \in \mathcal{H C}$.

The differential $d \Xi$ of $\Xi$ takes self-adjoints into self-adjoints, and takes any projection $P$ on $\mathcal{H}$ into "the number of particles with wave functions in $P$ FC." The creation operator $C(x)$ takes a vector $y \in \mathcal{L}$ representing a state with no $x$-particles (i.e., $\left.d \Xi P_{x}\right)$ has expectation value zero in this state, where $P_{x}$ is the projection of $\mathfrak{F}$ onto the one-dimensional subspace spanned by $x$ ) into a vector representing a state with precisely one $x$-particle.

An example is the trivial statistics in which $\mathcal{L}$ is the tensor algebra $K$ over $\mathfrak{T C}, \Xi=\Gamma$, and the $C(x)$ are defined as follows: an element $u$ of $K$ is in the domain of $C(x)$ if $\sum_{n} n^{(1 / 2)}\left|x \times u_{n}\right|^{2}$ is finite, and $C(x) u$ is then defined as $\sum_{n} n^{(1 / 2)} x \times u_{n}$. In these statistics, a set of identical particles can be given a significant order, and $C(x)$ as defined here corresponds to the creation of an $x$-particle in first position. The creation of an $x$-particle in last position would be represented by the operator $u \rightarrow \sum_{n} n^{(1 / 2)} u_{n} \times x$, while more generally the operator $C(x, \Lambda)$ representing the creation of an $x$-particle in a given position determined by the function $\Lambda$ is defined as follows:

$$
C(x, \Lambda) u=\sum_{n} n^{(1 / 2)} V^{(n+1)}(\pi(\Lambda(n)))\left(x \times u_{n}\right),
$$

where $\Lambda(n)$ is the positive integer between 1 and $n+1$ representing the position of the created particle relative to $n$ existing particles, while $\pi(r)$ is the 
permutation that consists of the transposition of 1 and $r$.

A more general type of quantum statistics, and in fact the only known type satisfying (1) and (2) as well as the condition that $d \Xi(P)$ have nonnegative integral eigenvalues when $P$ is a projection on $\mathcal{H}$, can be obtained from suitable subspaces $\mathcal{X}$ of $K$. If $\mathcal{X}$ is a "characteristic" subspace (see $\S 3$ ), it is left invariant by the $\Gamma(U)$, and there is induced on the quotient $K / \mathcal{X}$ a representation $\Xi$ defined by the equation $\Xi(U)(y+\mathcal{X})=\Gamma(U) y+\mathcal{X}$, satisfying the first condition. In order to satisfy the second condition a further specialization of $\mathcal{X}$ is necessary, and it is convenient to discuss first the notion of semi-graded operator, of which both the $\Gamma(U)$ and the $C(x, \Lambda)$ are examples.

Definition 2. An operator $T$ in a graded Hilbert space $\mathcal{L}=\sum_{n} \mathcal{L}_{n}$ is said to be semi-graded (relative to the given gradation) if its domain includes all the $\mathcal{L}_{n}$, and if $\mathcal{L}_{n}$ is mapped linearly and continuously by $T$ into $\sum_{k_{1}(n) \leqq m \leqq k_{2}(n)} \mathcal{L}_{m}$, where $k_{1}(m)$ and $k_{2}(m)$ go to infinity with $m$, while the domain consists specifically of all vectors $\sum_{n} x_{n}$ with $x_{n} \in \mathcal{L}_{n}$ and $\sum_{m}\left|\sum_{n} P_{m} T x_{n}\right|^{2}$ finite, where $P_{n}$ is the projection of $\mathcal{L}$ onto $\mathcal{L}_{n}$, such a vector being taken into $\sum_{m}\left(\sum_{n} P_{m} T x_{n}\right)$.

$A$ semi-graded operator is necessarily closed. To see this, let $T$ be semigraded, and let $x^{(r)}$ be in the domain of $T$ with $x^{(r)} \rightarrow x$ and $T x^{(r)} \rightarrow y$. Now $x$ is in the domain $D_{T}$ of $T$ if and only if $\sum_{m}\left|\sum_{n} P_{m} T x_{n}\right|^{2}$ is finite. But $\sum_{m<m_{1}}\left|\sum_{n} P_{m} T x_{n}\right|^{2}=\lim _{r} \sum_{m<m_{1}}\left|\sum_{n} P_{m} T x_{n}^{(r)}\right|^{2}$ and $\sum_{m}\left|\sum_{n} P_{m} T x_{n_{n}}^{(r)}\right|^{2}$ $=\left|T x^{(r)}\right|{ }^{2}$, which is bounded since $T x^{(r)} \rightarrow y$, so that $\sum_{m<m_{1}}\left|\sum_{n} P_{m} T x_{n}\right|^{2}$ is bounded $\left(m_{1}=1,2, \cdots\right)$, showing that $\sum_{m}\left|\sum_{n} P_{m} T x_{n}\right|^{2}$ is finite. To show that $T x=y$ it suffices to show that $P_{m} T x=P_{m} y$ for all $m$. By virtue of the bounded character of the action of $T$ on the $\mathcal{L}_{k}$ it is clear that $P_{m} T x=\lim _{r} P_{m} T x^{(r)}=P_{m} \lim _{r} T x^{(r)}=P_{m} y$.

In graphic terms, an operator is semi-graded with respect to a gradation if and only if it has a matrix representation relative to the decomposition of the Hilbert space given by the gradation $\mathcal{L}=\sum_{n} \mathcal{L}_{n}$ in which the matrix elements are bounded linear transformations from the $\mathcal{L}_{j}$ to the $\mathcal{L}_{k}$, and which vanish outside a monotone increasing band around the main diagonal which is of finite extent laterally and horizontally; and has the domain associated with this matrix representation, of vectors in the Hilbert space whose transforms under the matrix are sequences representing elements of the Hilbert space. From this it is clear that the semi-graded operators constitute a *algebra relative to the usual matrix operations. However, the matrix * need not correspond to the Hilbert space *, because the Hilbert space * will not necessarily be semi-graded. In the special case in which the semi-graded operator is the closure of its restriction to the algebraic elements (i.e., vectors of the form $\sum_{n} x_{n}$ with $x_{n} \in \mathcal{L}_{n}$ and $x_{n}=0$ for large $n$ ), the Hilbert space adjoint is again semi-graded. To prove this, let $T$ be such an operator, let $T^{\prime}$ denote the semi-graded (matrix) adjoint to $T$, and let $\mathcal{L}_{0}$ denote the set of all algebraic elements. Then $T^{*}$ is an extension of $T^{\prime}$, where $T^{*}$ denotes the usual Hilbert 
space adjoint. For this means that if $y \in \mathscr{D}_{T^{\prime}}$, then for all $x \in \mathcal{L}_{0},(T x, y)$ $=\left(x, T^{\prime} y\right)$, using the assumption that $T$ is the closure of its restriction to $\mathcal{L}_{0}$. This follows straightforwardly from the definition of the semi-graded adjoint $T^{\prime}$ as the unique semi-graded operator such that $(T x, y)=\left(x, T^{\prime} y\right)$ for all algebraic $x$ and $y$. To show conversely that $T^{\prime}$ is an extension of $T^{*}$, it must be shown that if $(T x, y)=\left(x, y^{\prime}\right)$ for all $x \in \mathcal{L}_{0}$, then $\sum_{m}\left|\sum_{n} P_{m} T^{\prime} y_{n}\right|^{2}$ is finite and $\sum_{m}\left(\sum_{n} P_{m} T^{\prime} y_{n}\right)=y^{\prime}$. Now $(T x, y)=\left(x, y^{\prime}\right)$ for $x \in \mathcal{L}_{n}$, which shows that $y_{m}^{\prime}=P_{m} T^{*} y^{(r)}$ for sufficiently large $r$, where $y^{(r)}=\sum_{m<r} y_{m}$. That is, $y_{m}^{\prime}=\sum_{n} P_{m} T^{\prime} y_{n}$ and $\sum_{m}\left|y_{m}^{\prime}\right|^{2}$ is finite, so that $y \in \mathcal{D}_{T^{\prime}}$ and $T^{\prime} y=\sum_{m}\left(\sum_{n} P_{m} T^{\prime} y_{n}\right)=\sum_{m} y_{m}^{\prime}=y^{\prime}$.

A semi-graded operator is said to leave invariant a graded subspace $\mathcal{N}=\sum_{n} \mathcal{N}_{n}$ in case it maps $\mathcal{N}_{n}$ into $\mathcal{N}$. An operator with this property determines a semi-graded "quotient" operator on the quotient space $\mathcal{L} / \mathcal{N}$ that is characterized as the semi-graded operator taking $x_{n}+\mathcal{N}$ into $T x_{n}+\mathcal{N}$ for $x_{n} \in \mathcal{L}_{n}(n=0,1,2, \cdots)$.

Returning now to the definition of suitable creation operators, if the characteristic subspace $\mathcal{X}$ of the tensor algebra $K$ is invariant under the creation operators $C(x, \Lambda)$, the quotient operators $C(x, \Lambda) / \mathcal{X}$ will transform states appropriately for a creation operator. This means that the second condition will be satisfied if $\mathcal{X}$ is a certain type of ideal, and it is readily seen that it suffices that the ideal be "completely characteristic" (see below). This is also close enough to being necessary that the subspaces invariant under the $C(x, \Lambda)$ can readily be investigated through the completely characteristic ideals, which are of more intrinsic mathematical interest, and the same as the completely characteristic ideals in the presence of simple physical side conditions relating either to symmetry or to maximality. In fact, a subspace that is characteristic and invariant under the canonical representations of the symmetric groups is readily seen to be completely characteristic, from the proof of Theorem 1. It is in the interest of obtaining an "elementary" quantum statistics, i.e. one into which no valid proper exclusion principle can be introduced, that maximality of the ideal is relevant; more precisely, it is appropriate to require that the ideal be maximal with respect to not containing all tensors from a certain rank onwards, as if the ideal contains such tensors, the corresponding statistics admits only a limited number of particles. Roughly speaking, in any quantum statistics the excluded states form an ideal in the tensor algebra over the one-particle space. It will be seen in the next section that the maximality assumption assures that the ideal be completely characteristic and in fact is either the symmetric or skew-symmetric ideal.

It is not difficult to see that the creation operators, both on $K$ and modulo invariant ideals, are the closures of their restrictions to the algebraic elements; this arises from the circumstance that they take elements of rank $n$ into elements of rank $n+1$. It results that the adjoints, which are the corre- 
sponding annihilation operators, are also semi-graded. In the cases of the symmetric and skew-symmetric ideals these relative creation and annihilation operators are nearly independent of the order function $\Lambda$ (precisely so in the symmetric case and within sign in the skew-symmetric case). It is useful then to introduce the Hermitian operators $P \mathcal{X}(x)$ and $Q \mathcal{S C}(x)$, which are respectively the closures of

$$
2^{-(1 / 2)}\left(\operatorname{Cgx}(x, e)+\operatorname{Cg}(x, e)^{*}\right) \text { and } i 2^{-(1 / 2)}\left(\operatorname{Cgx}(x, e)^{*}-\operatorname{Cg}(x, e)\right),
$$

where $e$ is the unit permutation and the subscript $\mathcal{H}$ signifies the corresponding quotient operators modulo $\mathfrak{X}$.

3. Determination of characteristic ideals. In this section the completely characteristic ideals in the algebra of covariant tensors over a Hilbert space are determined explicitly. By "characteristic" is meant invariant under all automorphisms of the algebra, while "completely characteristic" means invariant under all continuous linear operations commuting with the automorphisms as well. It follows that the only completely characteristic ideals that are maximal with respect to not containing all tensors from a certain rank onwards are the symmetric and skew-symmetric ideals.

TheOREM 1. A characteristic subspace $\mathcal{H}$ in the algebra of all covariant tensors over a complex Hilbert space $3 \mathcal{C}$ is graded and is completely characteristic if and only if the projection of $\mathfrak{H}_{n}$ (the space of $n$-tensors) onto the nth component $\mathcal{H}_{n}$ of $\mathcal{X}$ is the image under the canonical representation on $\mathfrak{H}_{n}$ of the symmetric group $\Sigma_{n}$ of degree $n$ of a central projection $q_{n}$ in the algebra of $\Sigma_{n}$. If the minimal such $q_{n}$ is employed (and $q_{n}$ is unique when HC is infinite-dimensional), such a subspace is an ideal if and only if whenever an irreducible representation of $\Sigma_{n}$ of type $\left(n_{1}, n_{2}, \cdots\right)$ occurs in the range of $q_{n}$ (here $n_{1} \geqq n_{2} \geqq \cdots, \sum_{k} n_{k}=n$, and each $n_{k}$ is a positive integer), then so also do the irreducible representations of types $\left(n_{1}+1, n_{2}, \cdots\right),\left(n_{1}, n_{2}+1, \cdots\right), \cdots$ (omitting those cases in which the coordinates of the type-vector fail to be nonincreasing) occur in the range of $q_{n+1}$.

Throughout the proof, which depends in part on a well-known theorem of Schur and on the branching law for irreducible representations of the symmetric group, the notation of the preceding sections is used.

Lemma: 1 . The projection of $K$ onto $\mathfrak{H}_{n}$ is in the ring $R$ generated by the $\Gamma(U), U$ unitary on $\mathcal{H C}$.

It is clear from the definition of $d \Gamma(A)$ for self-adjoint $A$ as the generator of the one-parameter group $\Gamma\left(e^{i t A}\right),-\infty<t<\infty$, that the spectral projections of $d \Gamma(A)$ are in $R$. Now the operator $d \Gamma(I)$ can be obtained as

$$
\lim _{t \rightarrow 0} t^{-1}\left(\Gamma\left(U_{t}\right)-I\right)
$$


where $U_{t}$ is the operation on $\mathscr{H C}$ of multiplication by $e^{i t}$. As $\Gamma_{n}\left(U_{t}\right)$ is easily seen to coincide with multiplication by $e^{i n t}, \mathfrak{F C}_{n}$ is in the domain of $d \Gamma(I)$ and $d \Gamma(I)$ coincides on it with multiplication by $n$. It follows that the projections of $K$ onto the $\mathfrak{H}_{n}$ are spectral projections of $d \Gamma(I)$ and so are in $R$.

The next lemma is dependent on the complex character of the Hilbert space. In the tensor algebra over a real Hilbert space the ideal generated by the tensors of the form $y \times y-(y, y) e$, where $e$ is the unit zero tensor, is characteristic but not graded.

Lemma 2. A characteristic subspace of $K$ is graded.

If $\mathcal{X}$ is a characteristic subspace, the projection $M$ with range $\mathscr{X}$ commutes with all elements of $R$, and in particular commutes with the projections $P_{n}$ of $K$ onto $\mathcal{H}_{n}(n=0,1,2, \cdots)$. It follows that the $P_{n} M$ are orthogonal projections and $M=\sum_{n} P_{n} M$, so that $\mathcal{X}$ is the direct sum of the ranges $\mathcal{X}_{n}$ of the $P_{n} M$; and obviously $\mathcal{X}_{n} \subset \mathcal{F}_{n}$.

Lemma 3. An operator on $\mathcal{F}_{n}$ that commutes with all $\Gamma_{n}(U)$ for $U$ unitary on HC is the image of an element of the algebra of $\Sigma_{n}$ under its canonical representation on $\mathfrak{H}_{n}$.

Let $\mathcal{D}$ be an arbitrary finite-dimensional subspace of $\mathcal{H}$, and put $\mathcal{D}_{n}$ for the $n$-fold tensor product of $\mathcal{D}$ with itself. Let $T$ be the operator in question, set $T(\mathcal{D})=P T P$, where $P(=P(\mathcal{D}))$ is the projection of $\mathfrak{H}_{n}$ onto $\mathscr{D}_{n}$, and put $T^{\prime}(\mathcal{D})$ for the contraction of $T(\mathcal{D})$ to $\mathscr{D}_{n}$. Now as $T$ commutes with all $\Gamma_{n}(U)$, it commutes in particular with those for which $U$ leaves $D$ invariant, and as $P$ also commutes with such $\Gamma_{n}(U)$, so also does $T(D)$. It follows that the operator $T^{\prime}(\mathcal{D})$ on $\mathscr{D}_{n}$ commutes with all $\Gamma_{n}(W, \mathcal{D})$ for $W$ a unitary on $\mathcal{D}$ to $\mathscr{D}$, where the notation emphasizes that $\mathcal{H}$ is being replaced by $\mathcal{D}$ in the formation of $\Gamma_{n}(W)$. Now a well-known theorem of Schur (see [11, p. 130]) implies that any such operator is in the algebra of the canonical representation on $\Phi_{n}$ of $\Sigma_{n}$.

Actually, as stated this theorem applies to tensor representations of the general linear rather than unitary group. However, the commutor of any tensor representation of one of the groups is the same as that of the corresponding representation of the other group. More generally, in fact, if $\Lambda$ is any uniformly continuous complex representation (on a complex Hilbert space) of the general linear group (over a complex Hilbert space), the uniformly closed enveloping algebra of $\Lambda$ is the same as that of the contraction of $\Lambda$ to the unitary subgroup. By a "complex" representation is meant one whose differential is complex-linear, where the differential $d \Lambda$ of $\Lambda$ is defined as the mapping from the bounded linear operators on the first Hilbert space $\mathcal{L}_{1}$ to those on the second Hilbert space $\mathcal{L}_{2}$ determined by the equation: $\exp (t d \Lambda(A))$ $=\Lambda\left(e^{t A}\right),-\infty<t<\infty$. The existence of such a map follows from the uniform continuity of the representation $\Lambda$, which is assumed to map into bounded 
operators, and to have the usual properties: $\Lambda(S T)=\Lambda(S) \Lambda(T)$ and $\Lambda\left(I_{1}\right)=I_{2}$, where $I_{j}$ is the identity on $\mathcal{L}_{j}(j=1,2)$. There is no difficulty in showing that this mapping is necessarily real-linear. The uniformly closed enveloping algebra of a representation is defined as the smallest uniformly closed algebra of bounded operators containing the operators in the range of the representation. The proof of the foregoing statement follows along familiar Lie-theoretic lines and is omitted.

In case $\mathfrak{H C}$ is finite-dimensional the lemma is a restatement of Schur's theorem. Suppose now that $\mathscr{H C}$ is infinite-dimensional. Let $V(\cdot, G)$ denote the canonical representation of $\Sigma_{n}$ on the $n$-fold tensor product $G_{n}$ of the Hilbert space $G$ with itself, or more inclusively the canonical representation of the algebra $A\left(\Sigma_{n}\right)$ of $\Sigma_{n}$. Then $T^{\prime}(\mathcal{D})=V(Y(\mathcal{D}), \mathcal{D})$ for some element $Y(\mathcal{D})$ of $\mathcal{A}\left(\Sigma_{n}\right)$ that is uniquely determined by $T^{\prime}(\mathcal{D})$ when the dimension of $\mathscr{D}$ exceeds $n$. Now if $\mathcal{D} \subset \mathcal{E}$, where $\varepsilon$ is a finite-dimensional subspace of $\mathcal{H}$, then $P(\mathcal{D}) \subset P(\mathcal{E})$, which means that $T^{\prime}(\mathcal{D})$ can be obtained from $T^{\prime}(\mathcal{E})$ by relativizing with respect to $\mathcal{D}_{n}$. This implies that $Y(\mathcal{D})$ is the corresponding relativization of $Y(\varepsilon)$. As $\Sigma_{n}$ leaves $\mathscr{D}_{n}$ invariant, it follows that $Y(\mathcal{D})$ and $Y(\mathcal{E})$ have the same action on $\mathcal{D}_{n}$, i. e. $V(Y(\mathcal{D}), \mathcal{D})=V(Y(\mathcal{E}), \mathcal{D})$. Therefore for $\mathcal{D}$ of dimension greater than $n, Y(\mathscr{D})$ has a constant value $Y$, and $T(\mathcal{D})$ $=P(D) V(Y, \mathfrak{F}) P(D)$. Now as a net indexed by the finite-dimensional subspaces $\mathcal{D}$, partially ordered by inclusion, $\{P(\mathcal{D})\}$ converges strongly to the identity operator on $\mathfrak{H}_{n}$, so that from its definition $\{T(D)\}$ converges strongly to $T$, while from the preceding equation it converges strongly to $V(Y, \mathfrak{F})$.

Lemma 4. $A$ graded subspace $\mathcal{H}=\sum_{n} \mathcal{X}_{n}$ is a right ideal if and only if $Q_{n} \times I \subset Q_{n+1}(n=0,1,2, \cdots)$, where $Q_{n}$ is the projection of $\mathcal{F C}_{n}$ onto $\mathcal{M}_{n}$.

If $\mathcal{X}$ is a right ideal, then for arbitrary $x \in \mathcal{F}$ and $y \in \mathcal{X}_{n}, y \times x \in \mathcal{X}_{n+1}$. This can be written as $Q_{n+1}\left(Q_{n} z \times x\right)=Q_{n} z \times x$, where $z$ is arbitrary in $\mathcal{H}_{n}$, or $Q_{n+1}\left(Q_{n} \times I\right)(z \times x)=\left(Q_{n} \times I\right)(z \times x)$. As the $z \times x$ span $\mathcal{H}_{n+1}$, it follows that $Q_{n+1}\left(Q_{n} \times I\right)=Q_{n} \times I$, or $Q_{n} \times I \subset Q_{n+1}$. The converse, that the latter inclusions imply that $\mathcal{H}$ is a right ideal, follows by reversing the preceding argument.

Proof of theorem. Let $\mathscr{X}$ be a completely characteristic subspace, so that by Lemma $2, \mathscr{X}=\sum_{n} \mathcal{X}_{n}$, with $\mathcal{X}_{n} \subset \mathcal{H}_{n}$. It follows from Lemma 3 that the projection $Q_{n}$ of $\mathscr{H C}_{n}$ onto $\mathcal{X}_{n}$ has the form $Q_{n}=V^{(n)}\left(q_{n}, \mathfrak{K}\right)$ for some element $q_{n}$ of $A\left(\Sigma_{n}\right)$. If $\mathfrak{H C}$ is infinite-dimensional (or of dimension greater than $n$ ), $V^{(n)}(\cdot, \mathfrak{F C})$ is an isomorphism, so that $q_{n}$ is a projection. If $\mathfrak{H C}$ is finite-dimensional, then as is well known there exists a central projection $c_{n}$ in $\mathcal{A}\left(\Sigma_{n}\right)$ such that $V^{(n)}\left(e-c_{n}, \mathfrak{F}\right)=0$, where $e$ is the group identity, while the contraction of $V^{(n)}(\cdot, \mathfrak{F})$ to the range of $c_{n}$ is an isomorphism. It follows that $q_{n}$ may be chosen as a projection in any case.

Lemma 3 now shows that the commutor $R_{n}^{\prime}$ of the ring $R_{n}$ generated by the $\Gamma_{n}(U)$ is the ring $S_{n}=V^{(n)}\left(\mathcal{C}\left(\Sigma_{n}\right), \mathfrak{H C}\right)$. The assumption that $\mathcal{X}$ is com- 
pletely characteristic means that the projection of $\mathfrak{H C}$ onto $\mathcal{X}$ is in the commutor of the commutor of $R$, i.e., in $R$ itself. As $R$ is the direct sum of the rings $R_{n}$, this means that $Q_{n}$ is in $R_{n}$. Now $R_{n}$ is the commutor of $S_{n}$, so it follows that $Q_{n}$ is central in $S_{n}$. It is easily seen that $q_{n}$ is therefore either central in $A\left(\Sigma_{n}\right)$ (e.g., if unique, as when $\mathcal{H}$ is infinite-dimensional) or may be so chosen. There is in fact a central $q_{n}$ that is minimal with respect to the property that $Q_{n}=V^{(n)}\left(q_{n}, \mathcal{F C}\right)$, and the notation " $q_{n}$ " will be reserved in the remainder of the proof for this projection.

Now suppose that $\mathcal{H}$ is an ideal. Then by Lemma $4, Q_{n} \times I \subset Q_{n+1}$. Now $\Sigma_{n}$ can be realized as a subgroup of $\Sigma_{n+1}$, by representing $\sum_{r}$ in general as the set of all permutations of the first $r$ positive integers, in the usual way, and $\mathcal{A}\left(\Sigma_{n}\right)$ is thereby a subalgebra of $\mathcal{A}\left(\Sigma_{n+1}\right)$. With this identification, $Q_{n} \times I$ $=V^{(n+1)}\left(q_{n}, \mathcal{H C}\right)$. As $\left.Q_{n+1}=V^{(n+1}\right)\left(q_{n+1}, \mathcal{H}\right)$ and as $V^{(n+1)}(\cdot, \mathcal{H C})$ is an isomorphism on the range of $c_{n}$, it follows from the foregoing inclusion that $q_{n} \leqq q_{n+1}$ in $\mathcal{A}\left(\Sigma_{n+1}\right)$. Now suppose that the conclusion of the theorem is violated: an irreducible representation of $\Sigma_{n}$ of type $\left(n_{1}, n_{2}, \cdots\right)$ is contained in the range of $q_{n}$, but there exists an irreducible representation of $\Sigma_{n+1}$ of the corresponding prescribed type that is not contained in the range of $q_{n+1}$. Now these corresponding prescribed types are precisely those which branch, on restriction to the subgroup $\Sigma_{n}$, into the given irreducible representation of $\Sigma_{n}$ (among other such representations) (see [12, pp. 342-343]). Hence there exists a projection $r_{n}$ that is minimal in $A\left(\Sigma_{n}\right)$ and contained in $q_{n}$, and a projection $r_{n+1}$ that is minimal in $\mathcal{A}\left(\Sigma_{n+1}\right)$ and not contained in $q_{n+1}$, while $r_{n} \leqq r_{n+1}$. As $r_{n+1}$ is minimal and $q_{n+1}$ is central, $r_{n+1} q_{n+1}=0$, so $r_{n+1} q_{n+1} q_{n}=0$, which implies by virtue of the equation $q_{n+1} q_{n}=q_{n}$ that $r_{n+1} q_{n}=0$. As $r_{n+1} \geqq r_{n}$, the last equation implies that $r_{n} q_{n}=0$, which is incompatible with $r_{n}$ being contained in $q_{n}$.

Now suppose conversely that the prescribed branching condition holds for the completely characteristic subspace $\mathcal{H}$. This means that if $r_{n}$ is a minimal projection in $\mathcal{A}\left(\Sigma_{n}\right)$ that is contained in $q_{n}$, and if $r_{n+1}$ is a minimal projection in $A\left(\Sigma_{n+1}\right)$ that contains $r_{n}$, then $q_{n+1}$ contains $r_{n+1}$ (actually its immediate significance is that $q_{n+1}$ contains a transform of $r_{n+1}$, but as $q_{n+1}$ is central it must then contain $r_{n+1}$ ). As $q_{n}$ is a sum of minimal projections, it follows that $q_{n+1} \geqq q_{n}$. Reversing the first part of the argument of the preceding paragraph, it results that $Q_{n} \times I \subset Q_{n+1}$, which implies that $\mathcal{X}$ is a right ideal. As it is completely characteristic, each component is invariant under the canonical representation of the symmetric group of corresponding degree, so that the ideal is two-sided.

It will next be shown that the only forms of quantum statistics that originate from a characteristic subspace of the covariant tensor algebra as in the preceding section and are "elementary" are the well-known ones $\left({ }^{4}\right)$.

(4) The result thereby provides a mathematical counterpart to the experimental fact that so far as is now known all elementary particles obey either Bose-Einstein or Fermi-Dirac statistics. 
COROLLARY 1.1. Let $\mathcal{X}$ be a subspace of the algebra of covariant tensors over a complex Hilbert space that is characteristic, invariant under the creation operators $C(x, \Lambda)$, and maximal among such subspaces with respect to not containing all tensors from a certain rank onwards. Then $\mathcal{X}$ is either the symmetric or the skew-symmetric ideal.

As $\mathcal{X}$ is characteristic, it is graded, say as $\mathcal{X}=\sum_{n} \mathcal{X}_{n}$. It is first shown that no $\mathcal{H}_{n}$ can contain both $S_{n} \mathfrak{H}_{n}$ and $A_{n} \mathfrak{F}_{n}$.

Let $\mathcal{N}$ be the smallest subspace of $K$ that contains both $S_{m} \mathfrak{H}_{m}$ and $A_{m} \mathfrak{H C}_{m}$ (for some fixed $m$ ) and is invariant under the $C(x, \Lambda)$. It is easily seen that $\mathcal{N}=\sum_{n \geqq m} \mathcal{N}_{n}$ with $\mathcal{N}_{m}=\left(S_{m}+A_{m}\right) \mathcal{H}_{m}$ and $\mathcal{N}_{n}$ equal to the span of the $C(x, \Lambda) u$, with $u \in \mathcal{N}_{n-1}$, when $n>m$. It is also easily seen that an element of $\mathcal{N}_{m+1}$ of the form $C(x, \Lambda) u$, with $u \in \mathcal{N}_{m}$, is taken by the canonical representation on $\mathfrak{F}_{m+1}$ of $\Sigma_{m+1}$ into another element of the same form, so that $\mathcal{N}_{m+1}$ is invariant under $\Sigma_{m+1}$. It follows in this fashion by induction that all the $\mathcal{N}_{n}$ are invariant under the corresponding $\Sigma_{n}$, so that $\mathcal{N}$ is a completely characteristic ideal. Now every irreducible representation of $\Sigma_{n}$ will branch eventually into either the identity or the parity representation of $\Sigma_{n}$ (among other representations of $\Sigma_{n}$ ). For in any partition of $n^{2}$ there must either be $n$ positive summands, in which case the partition can be obtained from the partition of $n, n=1+1$ $+\cdots+1$, by successive addition of unity to one of the summands in the partition, as in the branching law; or else one of the summands in the partition of $n^{2}$ must be greater than $n$, in which case it can be obtained from the trivial partition of $n, n=n$, in the same fashion. It follows from Theorem 1 that $\mathcal{N}$ contains all tensors of rank at least $n^{2}$.

Now if $\mathcal{X}$ contains no $S_{n} \mathfrak{H C}_{n}$ and also no $A_{n} \mathfrak{H}_{n}(n=0,1,2, \cdots)$, then $\mathscr{X}$ is orthogonal to all these subspaces and so is contained both in the symmetric and skew-symmetric ideals. Hence it may be supposed that there is an index $m$ for which $\mathcal{X}_{m}$ contains either $S_{m} \mathfrak{H}_{m}$ or $A_{m} \mathfrak{H C}_{m}$. Consider the former case-the argument is parallel for the latter. Then Theorem 1 shows that $\mathcal{X}_{n}$ contains $S_{n} \mathcal{H C}_{n}$ for all $n>m$, and so can contain $A_{n} \mathcal{F C}_{n}$ for no $n$. It follows that $\mathcal{X}_{n}$ is orthogonal to $A_{n} \mathcal{H C}_{n}$ for every $n$, which means that $\mathcal{X}_{n}$ is contained in the skew-symmetric ideal.

\section{The SYMMETRIC TENSOR ALGEBRA}

1. Summary of contents and notation. One of the main concerns of this part is a canonical equivalence between the space of "square-integrable functionals" over a real Hilbert,space and the symmetric tensor algebra over its complexification. This equivalence clarifies the structures of operators on both systems. An extension of features of the theory of integration and of harmonic analysis from finite to infinite-dimensional spaces is relevant to this equivalence and has intrinsic interest.

Integration over a Hilbert space is first treated. This is followed by an extension of the Plancherel theorem. The equivalence between the space of 
square-integrable functionals and the symmetric algebra is then set up and used to examine the structure of the $\Gamma(U)$, the $P(x)$ and $Q(x)$ (or rather of the quotient operators of these modulo the symmetric ideal), and the Plancherel-Wiener transform. Finally some further aspects of harmonic analysis on a Hilbert space are developed.

Throughout this part $\mathcal{F}^{\prime}$ denotes a real Hilbert space with complexification $\mathfrak{H C}$ (see below). As before, $K$ will denote the covariant tensor algebra over $\mathfrak{H}$, while $\mathfrak{H}$ will here denote the symmetric ideal, and $\mathcal{S}$ the quotient $g$-algebra, which will be called the symmetric tensor algebra over $\mathfrak{F}$, or for short the symmetric algebra. By "symmetric tensor" is meant an element of $K$ that is in the range of the symmetrization operator, but frequently "symmetric tensor" will be used for both notions, with the convention that the two concepts are identified via the isomorphism $u \leftrightarrow u+\mathcal{H}$, and that "multiplication," which will be indicated by juxtaposition, means multiplication in $\mathcal{S}$, unless otherwise stated.

As the full algebra $K$ occurs in this part only in connection with $\delta$, the subscript " $\mathcal{X}$ " will be omitted on quotient operators throughout the partso that $\Gamma(U), P(x)$, and $Q(x)$ denote the quotients of the corresponding original operators on $K$, will from this point be distinguished by a subscript " $o$ ". In situations in later papers where there appears to be some possibility of confusion, the quotient operators modulo the symmetric ideal will be further distinguished by a subscript " $s$," while those modulo the skewsymmetric ideal will be distinguished by a subscript " $a$. "

2. Integration with respect to weak distributions. Let $\mathcal{L}$ be a real topological linear space, and let $F$ be a weak distribution on $\mathcal{L}$, i.e., a linear map from the dual space $\mathcal{L}^{*}$ of continuous linear functionals on $\mathcal{L}$ to real-valued random variables. More precisely, "weak distribution" will signify an equivalence class of such maps, two of which are equivalent if for all finite sets of linear functionals in $\mathcal{L}^{*}$, the two sets of image random variables under the maps have the same joint distributions. It will be necessary to have some development of the theory of integration of functionals over $\mathcal{L}$ with respect to $F$. It is convenient to begin via the notion of probability algebra, which will be extended by making

Definition 3. A "real singular probability algebra" ( $\left.R, E^{\prime}\right)$ is a system composed of a real (associative) algebra $R$, together with a real linear functional $E^{\prime}$ on $R^{\prime}$ such that $\left(R, E^{\prime}\right)$ satisfies all the conditions for being a probability algebra in [9], except that the condition that $E^{\prime}\left(a^{2}\right)=0$ need not imply that $a^{2}=0$. A (complex) "singular probability algebra" is a system $(C, E)$ that is the complexification (as in [9]) of a real system $\left(R, E^{\prime}\right)$, or alternatively is defined through the following axioms: (1) $\mathcal{C}$ is an (associative) algebra over the complex number field with an adjoint operation $a \rightarrow a^{*}$, with the usual properties $\left[(a+b)^{*}=a^{*}+b^{*},(\lambda a)^{*}=\bar{\lambda} a^{*}\right.$ for complex $\lambda$, $\left.(a b)^{*}=a^{*} b^{*}, a^{* *}=a\right] ;(2)$ for any $a$ in $\mathcal{C}$, 


$$
E\left(a a^{*}\right) \geqq 0, \quad E\left(a^{*}\right)=\overline{E(a)},
$$

and there is a constant $\mu$ such that $E\left(b^{*} a b\right) \leqq \mu E\left(b^{*} b\right)$ for all elements $b$ of $\mathcal{C}$; (3) $C$ has a unit $e$ and $E(e)=1$.

Any singular probability algebra $(\mathcal{C}, E)$ canonically determines a probability algebra $\left(\mathcal{C}_{1}, E_{1}\right)$ by taking $\mathcal{C}_{1}$ to be the quotient of $\mathcal{C}$ modulo the ideal $\mathcal{F}$ of all elements $a \in \mathcal{C}$ such that $E\left(a^{*} a\right)=0$, and setting $E_{1}$ for the functional defined by the equation $E_{1}(a+\mathcal{F})=E_{1}(a)$.

By "tame function" on $\mathcal{L}$ will be meant a function $f$ on $\mathcal{L}$ of the form

$$
\left.f(u)=\phi(u \mathscr{S})^{*}\right),
$$

where $u \mathscr{H}$ * is the linear functional on the finite-dimensional subspace $\mathcal{X}^{*}$ of $\mathcal{L}^{*}$ determined by the equation

$$
u \mathscr{H} \mathcal{C}^{*}\left(x^{*}\right)=x^{*}(u)
$$$$
x^{*} \in \mathcal{X}^{*},
$$

and $\phi$ is a complex-valued Baire function on the dual $\mathcal{X}^{* *}$ of $\mathcal{X}^{*}$. Such a function is said to be "based on" the manifold $\mathcal{X}^{*}$. The set $\mathcal{C}$ of all bounded tame functions on $\mathcal{L}$ is easily seen to form an algebra over the complex numbers. Now note that in the case when $\mathcal{L}$ is finite-dimensional, the concept of weak distribution is equivalent to that of regular probability distribution, in the sense that there exists a unique regular probability measure $m$ on $\mathcal{L}$ such that $F$ can be taken to be (i. e. defines the same weak distribution as) the identity mapping from $\mathcal{L}^{*}$ to the measurable functions relative to $(\mathcal{L}, m)$ (or more precisely, the mapping thereby induced from $\mathcal{L}^{*}$ to the measurable functions modulo null functions relative to $(\mathcal{L}, m))$. It follows that, resuming now the discussion of the general case, there exists a unique regular probability distribution $m \mathscr{X}^{* *}$ on the dual of $\mathcal{X}^{*}$ such that the identity map from $\mathcal{X}^{*}$ into the measurable functions relative to $\left(\mathcal{H}^{* *}, m \mathcal{X}^{* *}\right)$ defines the same weak distribution as the contraction of $F$ to $\mathcal{X}^{*}$. Now setting $E(f)=\int \mathcal{X} \boldsymbol{N}^{* *} \phi d m \mathcal{X} * *$ for $f \in \mathcal{C}$, it follows in a straightforward fashion that $(\mathcal{C}, E)$ is a singular probability algebra that is determined by the weak distribution alone.

The (nonsingular) probability algebra of $(\mathcal{C}, E)$ determines (see [9]) an essentially unique probability space, in such a way that the elements of $\mathcal{C}$ can be identified with set of bounded random variables on the space that is weakly dense (i.e., dense in the topology induced by the correspondence with the dual of $L_{1}$, with the canonical weak topology on the dual), with $E$ corresponding to the expectation. Any random variable on this probability space will now be designated as a "measurable functional" on $\mathcal{L}$ relative to the given weak distribution, regardless of whether or not it has any type of representation as an actual numerical-valued function on $\mathcal{L}$. In fact, in general it will have no such representation, and the designation "functional" is to be understood in a generalized sense ("strict functional" will be used for an actual numerical-valued functional). Concepts from the theory of Lebesgue integra- 
tion, such as convergence in mean, convergence almost everywhere, $p$ th power integrability, which are independent of the representation of the probability space, will be applied to measurable functionals freely, and will also be applied to tame functionals with the convention that the tame (strict) functional is replaced in this connection by the corresponding element of the nonsingular probability algebra. The measurable functional corresponding to the tame functional $f$ will be designated as $\bar{f}$. The expectation of an integrable functional $f$ will also be written as $\int \mathcal{L} f(u) d F(u)$.

The circumstance that when $\mathcal{L}$ is infinite-dimensional the measurable functionals no longer have realizations as strict functionals as in the finitedimensional case materially alters the character of the relevant integration theory. In the present paper there is no occasion for a complete development of this theory but it may be helpful to suggest the nature of some of the differences from conventional theory by noting: (1) a sequence of tame functionals may converge everywhere on $\mathcal{L}$ without converging almost everywhere as measurable functionals, even when they are uniformly bounded; (2) a simple transformation on $\mathcal{L}$, such as $x \rightarrow x+a$, with $a$ a fixed vector, will in general induce no corresponding transformation on the measurable functionals.

On the other hand, the tame functionals based on a fixed finite-dimensional manifold $\mathfrak{X}^{*}$ are isomorphic, as a partially ordered algebra of measurable functionals with a partially-defined distinguished functional (the integral), to the measurable functions modulo null functions over $\left(\mathcal{X}^{* *}, m \mathcal{X} \boldsymbol{X}^{*}\right)$ by the canonical map $\tilde{f} \rightarrow \tilde{f} \mathscr{X} x^{* *}$, where $\tilde{f} \mathscr{X} \mathcal{C}^{* *}$ is the functional on $\mathscr{X}^{* *}$ determined by the equation $\tilde{f} \mathcal{F}^{* *}\left(u^{* *}\right)=f(u)$ for any $u$ such that $u^{*}(u)=u^{* *}\left(u^{*}\right)$ for all $u^{*}$ in $\mathscr{H}^{*}$.

In the special case of a Hilbert space there are well-known canonical isomorphisms between $\mathcal{L}_{,} \mathcal{L}^{*}$, and $\mathcal{L}^{* *}$. In this way both $\mathscr{H}^{*}$ and $\mathscr{H}^{* *}$ may be regarded as subsets of $\mathcal{L}^{*}$. In order to avoid unnecessary circumlecution such identifications as these will be made without further comment, in dealing with weak distributions on a Hilbert space.

It may be helpful to note that an alternative description of a weak distribution may be given in terms of the assignment of the regular probability measures $m \mathscr{X}^{* *}$ to the spaces $\mathcal{X}^{* *}$ for all finite-dimensional submanifolds $\mathcal{X}^{*}$ of $\mathcal{L}^{*}$. This assignment is such that if $\mathcal{H}^{*} \subset \mathcal{N}^{*}$, then $m \mathcal{N}^{* *}$ contracts to $m \mathscr{X} * *$ in the sense that the conditional distribution on the quotient space $\mathcal{N}^{* *} / \mathcal{Z}^{* *}$, where $Z^{* *}$ is the annihilator of $\mathcal{X}^{*}$ in $\mathcal{N}^{* *}$ of the distribution $m \mathcal{N}^{* *}$ (in the sense of the theory of probability) corresponds in the canonical isomorphism of $\mathcal{N}^{* *} / Z^{* *}$ with $\mathcal{X}^{* *}$ to $m \mathcal{H} \cdot *$. Conversely, given such an assignment of conventional probability distributions to the dual of each finite-dimensional subspace of $\mathcal{L}^{*}$, there exists a unique weak distribution on $\mathcal{L}$ from which it can be obtained in the preceding manner. In the case of a Hilbert space $\mathcal{H C}^{\prime}$ the family $\left\{m \mathcal{N}^{*}\right\}$ corresponds in the fashion described in the preceding 
paragraph to a normalized additive measure on the "tame" subsets of $\mathrm{JC}^{\prime}$, these being the subsets whose characteristic functions are tame, which is countably additive on the subsets that are based on a fixed finite-dimensional submanifold (i.e., whose characteristic functions are so based).

3. The Wiener transform. The main content of this section is an extension of the Plancherel theorem from the case of a finite-dimensional euclidean space to that of an arbitrary-dimensional real Hilbert space. It turns out to be appropriate for a variety of reasons to extend to general Hilbert spaces not ordinary Lebesgue measure, but a certain normalized measure equivalent to it in the sense of absolute continuity, namely the measure arising from a centered isotropic normal distribution. The change brought about by the necessity of accounting for the derivative of the one measure with respect to the other, while complicating some matters, is also the source of some notable simplifications.

By the (canonical) normal distribution on a real Hilbert space $\mathfrak{F C}^{\prime}$, with parameter $c$, is meant the unique weak distribution determined by a map $F$ from $\mathfrak{H C}^{\prime}$ into random variables, such that: (1) for any vector $u$ in $\mathcal{H C}^{\prime}, F(u)$ is normally distributed with zero mean and variance $c|u|^{2}$; (2) $F$ takes orthogonal vectors into independent random variables. By a polynomial on $\mathfrak{F C}^{\prime}$ is meant a tame functional on $\mathfrak{H C}^{\prime}$ that can be represented as a polynomial over a finite-dimensional subspace on which it is based. It is easily seen that any polynomial functional is square-integrable, and hence integrable, with respect to the normal distribution over $\mathfrak{H C}^{\prime}$.

The complexification $\mathfrak{F}$ of $\mathfrak{H C}^{\prime}$ may be defined as a complex Hilbert space with a distinguished conjugation $J$ leaving invariant a real subspace isomorphic to $\mathfrak{H}^{\prime}$, together with a fixed such isomorphism. To avoid circumlocution, $\mathcal{C}^{\prime}$ will be identified with a subspace of $\mathfrak{H}$, and it follows that every element of $\mathfrak{H C}$ can be uniquely expressed as $x+i y$, with $x$ and $y$ in $\mathfrak{F C}^{\prime}$, and that $J(x+i y)=x-i y$. The existence of the complexification is shown by the model of all pairs $(x, y)$ with $x$ and $y$ in $\mathcal{F}^{\prime}$, with the usual addition and real scalar multiplication, with multiplication by $i$ defined by the equation $i(x, y)$ $=(-y, x)$, and with the inner product defined by the equation $((x, y)$, $\left.\left(x^{\prime}, y^{\prime}\right)\right)=\left(x, x^{\prime}\right)+\left(y, y^{\prime}\right)+i\left[\left(y, x^{\prime}\right)-\left(x, y^{\prime}\right)\right]$. Now if $f^{\prime}$ is a polynomial on $\mathfrak{F C}^{\prime}$, it extends uniquely to a functional $f$ on $\mathfrak{F}$ that is given as a polynomial in the complex coordinates of a finite-dimensional subspace of $\mathfrak{H C}$ on which it is based. The map $f^{\prime} \rightarrow f$ is an algebraic isomorphism. The expression $f^{\prime}(x+i y)$ will be defined to be equal to $f(x+i y)$. It is easily seen that the complex conjugate of $f^{\prime}(x+i y)$ is the same as $\bar{f}^{\prime}(x-i y)$, where $\bar{f}^{\prime}$ is defined by the equation

$$
\bar{f}^{\prime}(x)=\overline{f(x)}, \quad \quad x \in \mathcal{H}^{\prime} .
$$

THEOREM 2. The mapping taking an arbitrary polynomial $f$ on a real Hilbert space $\mathrm{FC}^{\prime}$ into the polynomial $F$, where 


$$
F(y)=\int \mathcal{C}^{\prime} f\left(2^{(1 / 2)} x+i y\right) d N(x)
$$

and $N$ is the normal distribution on $\mathrm{HC}^{\prime}$, extends uniquely to a unitary transformation on $L_{2}\left(\mathfrak{H C}^{\prime}, N\right)$, whose inverse on the polynomials has the form $F \rightarrow f$, where $f(y)=\int \mathcal{F C}^{\prime} F\left(2^{1 / 2} x-i y\right) d N(x)\left(^{5}\right)$.

It should perhaps be recalled that $L_{2}\left(\mathcal{F C}^{\prime}, N\right)$ indicates the complex Hilbert space of all measurable square-integrable functionals over $\mathcal{H}^{\prime}$, with the inner product $(f, g)=\iint_{\mathcal{C}} f(x) \bar{g}(x) d N(x)$. The parameter of the normal distribution is not specified in the theorem as the result is independent of it. The proof of the theorem makes use of

Lemma 2.1. Let $A$ be an algebra of random variables on a probability space $P$ that determines the measure ring of the space and contains the identity function, and suppose that $A \subset L_{2}(P)$ and that $\mathcal{A}$ has a set of (algebraic) generators $\left\{f_{\mu}\right\}$ such that $\exp \left[\sum_{j=1}^{n} a_{j}\left|f_{\mu_{j}}(x)\right|\right]$ is a function in $L_{2}(P)$ for all non-negative $a_{j}$ and finite sets of indices $\mu_{1}, \cdots, \mu_{n}$. Then $\mathcal{A}$ is dense in $L_{2}(\mathbb{P})$.

It may be recalled that a determining set of random variables on a probability space is one such that every measurable set differs by a null set from a set in the smallest $\sigma$-ring with respect to which all the given random variables are measurable. Now if $k$ is an element of $L_{2}(P)$ that is orthogonal to $\mathcal{A}$, then $\int f_{1}^{n_{1}} f_{2}^{n_{2}} \cdots f_{r}^{n_{r}} \bar{k}=0$ for any finite set $f_{1}, f_{2}, \cdots, f_{r}$ of generators of $\mathcal{A}$ and non-negative integers $n_{1}, n_{2}, \cdots, n_{r}$, where a function raised to the power zero is here to designate the identity function. Multiplying by $\left(i a_{1}\right)^{n_{1}}\left(i a_{2}\right)^{n_{2}}$ $\cdots\left(i a_{r}\right)^{n_{r}} / \prod_{j-1}^{r}\left(n_{j} !\right)$, summing, and using the dominated convergence theorem, it follows that $\int \exp \left[i\left(a_{1} f_{1}+a_{2} f_{2}+\cdots+a_{r} f_{r}\right)\right] \bar{k}=0$. Putting $F\left(b_{1}, \cdots, b_{r}\right)$ for the integral of $k$ over the set $A$ in $\mathcal{P}$ where $\left(f_{1}(x), \cdots\right.$, $\left.f_{r}(x)\right) \in B, B$ being an arbitrary Borel set in $r$-space, this means that $\int \exp \left[i\left(a_{1} b_{1}+\cdots+a_{r} b_{r}\right)\right] d F\left(b_{1}, \cdots, b_{r}\right)=0$. As is well known, this implies that $F$ is a constant, which under the present circumstances must be zero. Thus for every set such as $A, \int_{A} f=0$. The sets of the form of $A$ constitute a ring of sets $B$, and $\int_{B} f=0$ for any such set. But the fact that the $f_{\mu}$ determine the measure ring of $P$ implies that an arbitrary measurable set in $P$ differs from a set of the form of $B$ by a set of arbitrarily small measure. Therefore $\int_{C} k=0$ for arbitrary measurable sets $C$, so that $k=0$.

Proof of theorem. Putting $W_{0}$ for the indicated map, there is no difficulty in verifying that $W_{0}$ is linear on the algebra $P$ of all polynomials over $\mathcal{H}^{\prime}$.

(5) In part, this theorem as well as preliminary work on integration over Hilbert space is an abstraction of work of Wiener [13] and Cameron and Martin [1] relating to Brownian motion and a rigorization of work of Feynman [4] relating to quantum field theory. Cf. also Friedrichs [5]. In terms of the conventional model for Brownian motion, the weak distribution $F$ defined by a Brownian motion $x(t)$ on $(-\infty, \infty)$ is given by: $F(f)=\int f(t) d x(t), f \in L_{2}(-\infty, \infty)$. 
To show that $W_{0}$ is an isometry, it suffices to show that $E(f \bar{g})=E(F \bar{G})$, where $F=W_{0} f$ and $G=W_{0} g$, for $f$ and $g$ ranging over a set $\mathscr{D}$ of functionals that span $P$ (algebraically). It is convenient to take $\mathcal{D}$ to consist of the set of all functionals $f$ on $H^{\prime}$ that have the form $f(x)=\left(x, f_{1}\right)^{n_{1}}\left(x, f_{2}\right)^{n_{2}} \cdots\left(x, f_{r}\right)^{n_{r}}$ for some finite orthonormal set $f_{1}, f_{2}, \cdots, f_{r}$ in $\mathcal{H C}^{\prime}$, the $n_{j}$ being non-negative integers (i.e. $\mathcal{D}$ is the set of all monomials in all possible orthonormal coordinate systems). It is no essential loss of generality to take the coordinate system relative to which $g$ has a monomial expression to be the same as that involved in the expression of $f$ as a monomial. Thus it suffices to take the case of $f(x)=\hat{x}_{1}^{m_{1}} \hat{x}_{2}^{m_{2}} \cdots \dot{x}_{r}^{m_{r}}$ and $g(x)=\hat{x}_{1}^{n_{1}} \hat{x}_{2}^{n_{2}} \cdots \hat{x}_{r}^{n_{r}}$, where $\hat{x}_{j}$ denotes the functional defined by the equation $\hat{x}_{j}(x)=\left(x, f_{j}\right)$. Now denoting the expectation of a functional of several variables on $\mathfrak{H C}^{\prime}$ when all but one of the variables, say $x$, is held fixed, so that roughly speaking the integration is with respect to $x$, by the notation $E_{x}$, it is easily seen that

$F(y)=E_{x}\left[\left(\hat{x}_{1}\left(2^{(1 / 2)} x+i y\right)\right)^{m_{1}}\right] E_{x}\left[\left(\hat{x}_{2}\left(2^{(1 / 2)} x+i y\right)\right)^{m 2}\right] \cdots E_{x}\left[\left(\hat{x}_{r}\left(2^{(1 / 2)} x+i y\right)\right)^{m_{r}}\right]$,

using the fact that the expectation of a product of independent functions is the product of the respective expectations. There is a similar expression for $G(y)$, and it results that

$$
\begin{array}{r}
E(F \bar{G})=E_{y}\left\{E_{x}\left[\left(\hat{x}_{1}\left(2^{(1 / 2)} x+i y\right)\right)^{m_{1}}\right] E_{x}\left[\left(\hat{x}_{1}\left(2^{(1 / 2)} x-i y\right)\right)^{n_{1}}\right]\right\} \\
\cdots E_{y}\left\{E_{x}\left[\left(\hat{x}_{r}\left(2^{(1 / 2)} x+i y\right)\right)^{m_{r}}\right] E_{x}\left[\left(\hat{x}_{r}\left(2^{(1 / 2)} x-i y\right)^{n_{r}}\right]\right\}\right.
\end{array}
$$

using independence once more. On the other hand, $E(f \bar{g})=E\left(\hat{x}_{1}^{m_{1}+n_{1}}\right) \ldots$ $E\left(\hat{x}_{r}^{m_{r}+n_{r}}\right)$. It follows that it is sufficient to show that

$$
E_{y} E_{x}\left[\left(\hat{x}_{1}\left(2^{(1 / 2)} x+i y\right)\right)^{m_{1}}\right] E_{x}\left[\left(\hat{x}\left(2^{(1 / 2)} x-i y\right)\right)^{n_{1}}\right]=E\left(\hat{x}_{1}^{m_{1}+n_{1}}\right)
$$

in order to conclude that $W_{0}$ is isometric. As all the functions involved in the last equation are based on the one-dimensional manifold'spanned by $f_{1}$, the equation reduces to the equality of two integrals over one-dimensional Hilbert spaces. It is in fact easily seen to be equivalent to the statement that if $x$ and $y$ are independently distributed normal random variables with zero means and variances $c$, then

$E\left(x^{m+n}\right)=E_{y}\left\{E_{x}\left[\left(2^{(1 / 2)} x+i y\right)^{m}\right] E_{x}\left[\left(2^{(1 / 2)} x-i y\right)\right]\right\} \quad(m, n=0,1,2, \cdots)$,

where as usual " $E$ " signifies expectation. To prove this it is enough to show that the sums over $m$ and $n$ of the products of either side with $s^{m} t^{n} / m ! n !$ are finite and equal, for all real $s$ and $t$. Using dominated convergence there is obtained in this way on the left side the expression

$$
(2 \pi c)^{-(1 / 2)} \int \exp \left[(s+t) x-x^{2} /(2 c)\right] d x,
$$

and on the right the expression 


$$
\begin{aligned}
& (2 \pi c)^{-(3 / 2)} \iint \exp \left[s\left(2^{(1 / 2)} x+i y\right)-x^{2} /(2 c)\right] d x \\
& \qquad \exp \left[t\left(2^{(1 / 2)} x-i y\right)-x^{2} /(2 c)\right] d x \exp \left[-y^{2} / 2 c\right] d y .
\end{aligned}
$$

Using the well-known fact that $(2 \pi)^{-(1 / 2)} \int \exp \left(-x^{2} / 2\right)=1$ and a familiar method in complex variables, it is easy to conclude that for any complex $v,(2 \pi c)^{-(1 / 2)} \int \exp \left[v x-x^{2} /(2 c)\right] d x=\exp \left(c v^{2} / 2\right)$, and from this formula the equality of the two expressions follows readily by direct computation.

That $W_{0}$ is onto $P$ follows once the given expression for $W_{0}^{-1}$ is established, and by an argument similar to that used in the preceding paragraph, it is sufficient to obtain the formula for the case of the functionals $f(x)=x^{n}$ on a one-dimensional Hilbert space which can be identified with the real numbers. That is, it suffices to show that if $F=W_{0} f$, where $f(x)=x^{n}$, then $f(y)=$ $E_{x}\left[F\left(2^{(1 / 2)} x-i y\right)\right]$. Now $E_{x}\left[F\left(2^{(1 / 2)} x-i y\right)\right]=E_{x}\left\{E_{u}\left[f\left(2^{(1 / 2)} u+y+i 2^{(1 / 2)} x\right)\right]\right\}$, so that the desired equation can be expressed as

$$
\begin{array}{r}
y^{n}=(2 \pi c)^{-1} \int\left[\int\left(2^{(1 / 2)} u+y+i 2^{(1 / 2)} x\right)^{n} \exp \left(-u^{2} / 2 c\right) d u\right] \\
\exp \left(-x^{2} / 2 c\right) d x \\
(n=0,1,2, \cdots)
\end{array}
$$

To verify these equations it suffices to show that the two sides agree after multiplication by $s^{n} / n$ ! and summation over $n$. On the left this yields $e^{s y}$, while on the right, using dominated convergence, there results

$$
(2 \pi c)^{-1} \iint \exp \left[s\left(2^{(1 / 2)} u+y+i 2^{(1 / 2)} x\right)-\left(u^{2}+x^{2}\right) /(2 c)\right] d u d x,
$$

which can be readily evaluated as $e^{s y}$ also.

Since Lemma 2.1 shows that $P$ is dense in $L_{2}\left(\mathcal{F}^{\prime}, N\right)$, the proof is complete.

Comment. It may be illuminating as well as of intrinsic interest to show how the conventional form of the Plancherel theorem can be derived from Theorem 3. It may be supposed that the Hilbert space $\mathfrak{3 C}^{\prime}$ is one-dimensional, as a similar argument applies to any finite-dimensional space. There is then no essential loss of generality in taking $\mathcal{F C}^{\prime}$ to be the reals with the usual inner product, and $P$ then becomes the algebra of all complex polynomials over the real space $\mathcal{H C}^{\prime}$, with the distinguished functional $E$ on $\mathcal{P}$ expressible in the form $E(f)=(2 \pi c)^{-(1 / 2)} \int f(x) e^{-x^{2} / 2 c} d x$. The map $Z_{0}: f(x) \rightarrow f(x) \exp \left(-x^{2} /(4 c)\right)$ is thus isometric from $\mathcal{P}$ to $L_{2}(-\infty, \infty)$, relative to the usual inner product on the latter space. That this isometry extends to a unitary correspondence $Z$ between $L_{2}\left(\mathcal{H C}^{\prime}, N\right)$ and $L_{2}(-\infty, \infty)$ follows from Lemma 2.1, which applied to 
the ring of polynomials on the probability space $(-\infty, \infty)$ with the element of probability $(2 \pi c)^{-(1 / 2)} \exp \left(-x^{2} /(2 c)\right)$ shows the density of functions of the form $f(x) \exp \left(-x^{2} /(4 c)\right)$ in $L_{2}(-\infty, \infty)$. Putting $W_{c}$ for the unitary transformation on $L_{2}\left(\mathcal{H}^{\prime}, N\right)$ defined by Theorem 2 , the unitary transform $Z W_{c} Z^{-1}$ of $W_{c}$ is the conventional Fourier-Plancherel transform, apart from normalization constants.

To prove this it suffices to show that the two operators agree on functions of the form $g(x)=f(x) \exp \left(-x^{2} /(4 c)\right)$. Now direct computation involving a simple change of variable shows that this function is taken by $Z W_{c} Z^{-1}$ into the function of $y,(4 \pi c)^{-(1 / 2)} \int \exp (i u y / 2 c) g(u) d u$.

The term "Fourier-Plancherel transform" will be reserved for the conventional transform, while the present transform from $L_{2}\left(\mathrm{HC}^{\prime}, N\right)$ to $L_{2}\left(\mathrm{HC}^{\prime}, N\right)$, which in the finite-dimensional case is canonically unitarily equivalent to the Fourier-Plancherel transform, will here be called the "Wiener" transform.

4. Equivalence of $L_{2}\left(\mathcal{F C}^{\prime}, N\right)$ and the symmetric algebra over $\mathcal{H}$. Let the complexification of the real Hilbert space $\mathfrak{H C}^{\prime}$ be designated $\mathfrak{H}$. The symmetric tensor algebra $S$ over $\mathfrak{H C}$ has its algebraic subalgebra $S_{1}=K_{1} / \mathscr{H}$, where $K_{1}$ is the set of all algebraic elements in $K$, canonically isomorphic to the algebra $\mathcal{P}$ of polynomials over $\mathcal{K}^{\prime}$. To set up this isomorphism, which will be called the canonical elementary map between the polynomials over $\mathfrak{H C}^{\prime}$ and the algebraic symmetric tensors over $\mathcal{H}$, and designated $A$, let $u$ be an arbitrary algebraic symmetric tensor (in the strict sense, i.e. an element of $K$ rather than of a quotient ring of $K$ ). A functional $f_{u}$ on $\mathfrak{F C}^{\prime}$ is determined by the equation $f_{u}(x)=\sum_{n}\left(x^{n}, u_{n}\right)$, with $x^{0}$ interpreted as the unit in $\mathcal{H}_{0}$, where $u_{n}$ denotes the component in $\mathfrak{H C}_{n}$ of $u$. The map $A: u+\mathcal{X} \rightarrow f_{u}$ is readily seen to have the stated properties.

Actually it is a different map between the polynomials and the algebraic symmetric tensors that is significant in the following. While the latter map is not an algebraic isomorphism, it extends, as $A$ does not, to a unitary map of $S$ onto $L_{2}\left(\mathcal{H}^{\prime}\right)\left({ }^{6}\right)$. The completely characteristic operator $Z$ on $S$ which takes a tensor $u$ of rank $n$ into $u /\left((2 c)^{n}(n !)\right)^{(1 / 2)}$ is relevant to the map, which is described in

THEOREм 3. The map $W_{c} A Z$ from the algebraic symmetric tensors over the complexification $\mathfrak{H}^{\mathrm{C}}$ of the real Hilbert space $\mathfrak{F C}^{\prime}$ to the functionals on $\mathfrak{H C}^{\prime}$ extends uniquely to a unitary transformation of the symmetric tensor algebra over $\mathfrak{H C}$

(6) Mappings between sets of functions that can be formulated as special instances of this map occur both in quantum mechanics and in the theory of Brownian motion. Concerning the quantum-mechanical situation, see the treatment in [3] of the representation of a boson field in terms of an assembly of harmonic oscillators and that in [5] giving a (formal) spectral resolution of the $Q\left(x_{n}\right)$. For the situation in the theory of Brownian motion, which is mathematically rigorous, see [6] and [13]. Actually an abstract Hilbert space suffices for the purposes of [6], and the work there can be simplified by replacing the one-parameter family of Hermite functions by a conventional system. 
onto the space of square-integrable functionals over $H^{\prime}$, relative to the normal distribution with parameter $2 c$.

The transformation $W_{c}$ is the Plancherel-Wiener transform with parameter $c$ defined by Theorem 2, but the integration over $\mathfrak{H C}^{\prime}$ relevant to the present theorem is with respect to the normal distribution with parameter $2 c$. This is legitimate since as used here, $W_{c}$ acts only on the algebraic elements which are common to all the spaces $L_{2}\left(\mathcal{F C}^{\prime}, c^{\prime}\right)$, regardless of the parameter $c^{\prime}$ (where $L_{2}\left(\mathcal{F C}^{\prime}, c^{\prime}\right)$ stands for $L_{2}\left(\mathcal{F C}^{\prime}, N\right)$ with $N$ the normal distribution of parameter $\left.c^{\prime}\right)$.

To prove the theorem it suffices to show that $W_{c} A Z$ takes an orthonormal basis of $S$ onto an orthonormal basis for $L_{2}\left(\mathfrak{F C}^{\prime}, 2 c\right)$. Let the indexed family $\left\{x_{\mu}\right\}$ be an orthonormal basis for $\mathcal{H}^{\prime}$; then an orthonormal basis for $\mathcal{S}$ is given by the algebraic elements $x_{n(\cdot)}$, where $n(\cdot)$ is a function on the indices $\mu$ to the non-negative integers with $n=\sum_{\mu} n(\mu)$ finite, defined by the equation $x_{n(\cdot)}=\left(n ! / \prod_{\mu} n(\mu) !\right)^{(1 / 2)} \prod_{\mu} x^{n(\mu)}$, in which formula symbols with zero exponents are to be interpreted as unity and deleted. The given mapping takes $x_{n(\cdot)}$ into $W_{c}\left(\prod_{\mu} k_{\mu, n(\mu)}\right)$, where $k_{\mu, m}$ is the functional given by the equation $k_{\mu, m}(x)=\left(x, x_{\mu}\right)^{m} r_{m}(2 c)$, with $r_{m}(c)=\left(m ! c^{m}\right)^{-(1 / 2)}$; when $m=0, k_{\mu, m}$ is defined to be identically unity, and may be deleted in the product defining $x_{n(.)}$. Because of the independence of the $k_{\mu, 1}$ for different $\mu$, this expression for the image of $x_{n(\cdot)}$ can be written as $\prod_{\mu} W_{c} k_{\mu, n(\mu)}$, and the orthonormality of these images would follow if it were known that the $W_{c} k_{\mu, m}$ for $\mu$ fixed and $m=0,1,2, \cdots$ were orthonormal. Now the $k_{\mu, m}$ for fixed $\mu$ all are based on the one-dimensional subspace determined by $x_{\mu}$, as are their Wiener transforms, so that the latter orthonormality reduces to an assertion about integration over one-dimensional Hilbert spaces and specifically to the following: if $\mathfrak{H C}^{\prime}$ is a one-dimensional Hilbert space that is identified with the real line with the usual inner product $(a, b)=a b$, and if $k_{m}$ denotes the function defined by the equation $k_{m}(x)=r_{m}(2 c) x^{m}(m=0,1,2, \cdots)$, then the $W_{c} k_{m}$ are orthonormal in $L_{2}\left(\mathcal{H C}^{\prime}, 2 c\right)$. (It may be of interest to remark that the Wiener transforms of the $x^{m}$ are the Hermite polynomials, apart from constant factor and a change of scale depending on $c$.)

Now denoting inner products in $L_{2}(\mathfrak{F}, 2 c)$ by the subscript " $2 c$," the expression

$$
\sum_{m, n=0}^{\infty}\left(W_{c} k_{m}, W_{c} k_{n}\right)_{2 c} s^{m} t^{n} /\left(m ! n ! r_{m}(2 c) r_{n}(2 c)\right)
$$

can be evaluated by the dominated convergence theorem as $\left(W_{c} p, W_{c} q\right)_{2 c}$, where $p(x)=e^{s x}$ and $q(x)=e^{t x}$, which inner product is also seen to be finite. The formula given in the preceding section for the expectation of $e^{v x}$ shows in fact that $\left(W_{c} p, W_{c} q\right)_{2 c}=e^{2 c a t}$. It follows that the $W_{c}\left(k_{m}\right)$ are orthogonal for different $m$, and that $\left|W_{c}\left(k_{m}\right)\right|_{2 c}=\left(r_{m}(2 c)\right)^{-1}$, i.e. the $W_{c} k_{m}$ are normalized. 
It remains only to show that the transforms of the $x_{(\cdot)}$ span $L_{2}\left(\mathcal{G C}^{\prime}, 2 c\right)$. Now if $f$ is arbitrary in $P, Z^{-1} A^{-1} W_{c}^{-1} f$ is an algebraic element of $S$ which maps into $f$. Thus the transforms of the $x_{(\cdot)}$ span $P$ in the algebraic sense, and as $P$ is dense in $L_{2}\left(\mathcal{F C}^{\prime}, 2 c\right)$, the proof is complete.

The unitary transformation defined by Theorem 3 will be called the duality transform, as it takes a representation in which the particle aspect is explicit into one in which the wave aspect is paramount (i.e. the spectral resolutions of the relevant operators is made explicit; cf. Corollary 3.4). The map just defined from $S$ onto $L_{2}(\mathcal{H C}, c)$ (i. e. with the parameter halved) will be designated as $D_{c}$, or simply as " $D$ " when the parameter is clear from the context; $r_{m}(c)$ will similarly be denoted as " $r_{m} . "$

A unitary transformation $U$ on $\mathcal{H}$ is "real" if it leaves $\mathcal{H}$ " invariant, or equivalently if it is left invariant by the canonical conjugation.

CoROllary 3.1. If $U$ is a real unitary on $\mathfrak{H C}$, then $D_{c} \Gamma(U) D_{c}^{-1}$ acts on the polynomial algebra $P$ as: $f(x) \rightarrow f\left(U^{*} x\right)\left({ }^{7}\right)$.

As both $D_{c} \Gamma(U) \dot{D}_{c}^{-1}$ and the operation $f(x) \rightarrow f\left(U^{*} x\right)$ are isometric, it suffices to check that the two operators agree on the algebraic elements. Note first that $\left(A \Gamma(U) A^{-1} f\right)(x)=f\left(U^{*} x\right)$ for $f$ in $P$. It is sufficient to check this for the case in which $f$ is a monomial in some coordinate system, say $f(x)$ $=\left(x, x_{1}\right)^{n_{1}} \cdots\left(x, x_{r}\right)^{n_{r}}$, and then $A^{-1} f=x_{1}^{n_{1}} \cdots x_{r}^{n_{r}}, \Gamma(U) A^{-1} f=\left(U x_{1}\right)^{n_{1}}$ $\cdots\left(U x_{r}\right)^{n_{r}}$, so that $A \Gamma(U) A^{-1} f=g$, where $g(x)=\left(x, U x_{1}\right)^{n_{1}} \cdots\left(x, U x_{r}\right)^{n_{r}}$ $=\left(U^{*} x, x_{1}\right)^{n_{1}} \ldots\left(U^{*} x, x_{r}\right)^{n_{r}}=f\left(U^{*} x\right)$. Now because of the readily established invariant character of the algebra $P$ and of the Wiener transform under the real unitaries, $D_{c}$ commutes on $P$ with the operator $f(x) \rightarrow f\left(U^{*} x\right)$. Thus if $v$ is a symmetric tensor of rank $n,\left(D_{c} \Gamma(U) D_{c}^{-1}\right)\left(D_{c} v\right)(x)=\left(D_{c} \Gamma(U) v\right)(x)$ $=r_{n} W_{c / 2} A \Gamma(U) A^{-1}(A v)(x)=\left(W_{c / 2} A v\right)\left(U^{*} x\right)=\left(D_{c} v\right)\left(U^{*} x\right)$. As the $W v$ span the algebraic elements in $L_{2}\left(\mathcal{F C}^{\prime}, c\right)$, the corollary follows.

Corollary 3.2. $D_{c} \Gamma\left(i I_{\mathcal{H}}\right) D_{c}^{-1}=W_{c}$.

It is convenient to prove the corollary in the form $D_{c} \Gamma(i I)=W_{c} D_{c}$, putting $I$ simply for $I$ Ke. It suffices to show that these two operators agree on vectors of the form $x_{1}^{n_{1}} \cdots x_{r}^{n_{r}}$. Now $D_{c}\left(\dot{x}_{1}^{n_{1}} \cdots x_{r}^{n_{r}}\right)=r_{n} \prod_{j} W_{c / 2} k_{j}$, where $k_{j}(x)$ $=\left(x, x_{j}\right)^{n_{j}}$, so that $W_{c} D_{c}\left(x_{1}^{n_{1}} \cdots x_{r}^{n_{r}}\right)=r_{n} \prod_{j} W_{c} W_{c / 2} k_{j}$ by direct computation. On the other hand, by a similar computation, $D_{c} \Gamma(i I)\left(x_{1}^{n_{1}} \cdots x_{r}^{n_{r}}\right)$ $=i^{n} r_{n} \prod_{j} W_{c / 2} k_{j}$, so that it suffices to show that for a one-dimensional space ${ }^{\prime} \mathcal{C}^{\prime}, i^{n} W_{c / 2} k_{n}=W_{c} W_{c / 2} k_{n}$, where $k_{n}(x)=x^{n}$ here, making the usual identification of a one-dimensional real Hilbert space with the real line.

Now $\sum_{n=0}^{\infty}\left(i^{n} s^{n} / n !\right)\left(W_{c / 2} k_{n}\right)(y)=(2 \pi c)^{-(1 / 2)} \int \exp \left[i s x-(x-i y)^{2} /(4 c)\right] d x$ by the dominated convergence theorem, writing the Wiener transform in the form $\left(W_{c} f\right)(y)=(4 \pi c)^{-(1 / 2)} \int f(x)$ exp $\left[-(x-i y)^{2} / 4 c\right] d x$ for elements $f$ of $P$,

( ${ }^{7}$ This result can be regarded as a mathematical form for certain notions of Feynman concerning the connection between classical motions and quantum field motions. 
which yields the equation $\sum_{n}\left(i^{n} s^{n} / n !\right)\left(W_{c / 2} k_{n}\right)(y)=\exp \left[\left(c s^{2}\right) / 2+i s y\right]$. Making a change of variable in this equation, applying $W_{c}$ to both sides, and using the fact that $W_{c}$ is unitary as well as a simple convergence argument (in $\left.L_{2}\left(\mathcal{F C}^{\prime}, c\right)\right), \sum_{n}\left(s^{n} / n !\right)\left(W_{c} W_{c / 2} k_{n}\right)(y)$ is found to be the result of applying $W_{c}$ to the functional $\exp \left[-\left(c s^{2}\right) / 2+s y\right]$, which can be readily evaluated as $\exp \left[(1 / 2) c s^{2}+i s y\right]$.

Corollary 3.3. $W_{c}^{-1} P(x) W_{c}=Q(x)$ and $W_{c}^{-1} Q(x) W_{c}=-P(x)$.

The preceding corollary shows that $W_{c}^{-1} C(x) W_{c}=-i C(x)$, and as $2 C(x)$ $=P(x)+i Q(x)$, the present corollary follows directly.

CoRollary 3.4. For any $x$ in $\mathfrak{H C}^{\prime}, D_{c} Q(x) D_{c}^{-1}$ is the operation of multiplication by the functional $f_{x} /(2 c)^{(1 / 2)}$, where $f_{x}(y)=(y, x)\left({ }^{8}\right)$.

It should be recalled that the operation of multiplication by a measurable function on $L_{2}$ over the measure space has as its domain all elements of $L_{2}$ for which the (pointwise) product is again in $L_{2}$. Thus the domain $\mathscr{D}$ of multiplication $M$ by $f_{x} /(2 c)^{(1 / 2)}$ contains $P$, and $(M k)(y)=f_{x}(y) k(y) /(2 c)^{(1 / 2)}$, for $k \in \mathcal{P}$, but $\mathcal{D}$ may be more inclusive than $\mathscr{P}$; and due to the circumstance that the set over which the relevant measure is defined need not be $\mathcal{F C}^{\prime}$ itself, there need be no simple pointwise formulation for the action of $M$ on an element of $\mathcal{D}-\mathcal{P}$, in terms of points of $\mathfrak{F C}^{\prime}$. However, the action of $M$ on $\mathscr{D}$ can be derived from its action on $P$ by means of the fact that $M$ is the closure of its restriction to $P$. This follows from

LeMma 3.4.1. Let $\phi$ be a real measurable function on a measure space $\mathbb{B}$, and let $\mathcal{E}$ be a dense domain in $L_{2}(B)$ that is contained in the domain of the operation $M$ of multiplication by $\phi$, is invariant under $M$, and such that $\exp \left(s \phi^{a}\right) f \in L_{2}(B)$, where $\phi^{a}$ denotes the function defined by the equation $\phi^{a}(x)=|\phi(x)|$, for all sufficiently small positive $s$ and $f \in \mathcal{E}$. Then the contraction of $M$ to $\mathcal{E}$ is essentially self-adjoint.

For otherwise there exists a nonzero element $g$ of $L_{2}(B)$ such that either $(\phi f+i f, g)=0$ for all $f$ in $\varepsilon$, or $(\phi f-i f, g)=0$ for all $f$ in $\varepsilon$. As the same proof applies to both cases, take, say, the latter one. Then $\left(\phi^{k} f, g\right)=i^{k}(f, g)$ for $k=0,1,2, \cdots$ (interpreting $\phi^{0}$ as 1 ), whence $\sum_{k}\left(\phi^{k} f, g\right)(i s)^{k} / k !=(f, g)$ $\cdot \sum_{k}(-s)^{k} / k$ !. The condition of the lemma allows the interchange of summation and integration for sufficiently small positive $s$ and it results that

$$
\int e^{i s \phi} f \bar{g}=e^{-s} \int f \bar{g} \text {. }
$$

As $\varepsilon$ is dense, it is easy to conclude that this equation holds for all $f$ in $L_{2}(B)$.

$\left.{ }^{8}\right)$ Thus $\mathcal{H}^{\prime}$ may be taken as the medium of the waves representing the oscillatory aspect of a boson field (of particles with wave functions in $\mathcal{H C}$ ). 
Hence $e^{i \phi s} \bar{g}=e^{-s} \bar{g}$ almost everywhere, for all sufficiently small positive $s$. It follows readily that $i \phi+1$ vanishes almost everywhere on the set where $g$ is nonzero, but as $\phi$ is real-valued, this implies that $g=0$.

Completion of proof of corollary. It suffices now to show that $D Q(x) D^{-1}$ agrees with multiplication by the given functional on the domain $\varepsilon$ of all functionals that are images under the canonical elementary isomorphism $A$ of elements of the subalgebra of $S$ generated algebraically by the $\left\{x_{\mu}\right\}$, these being an indexed maximal orthonormal set in $\mathrm{JC}^{\prime}$ with $x=x_{v}$. (It is clearly no essential loss of generality to take $x$ to be normalized.) For as $Q(x)$ is closed and hermitian, so also is $D Q(x) D^{-1}$, and to show that a closed hermitian operator $T_{1}$ is the same as a self-adjoint operator $T_{2}$, it suffices to show that $T_{1}$ and $T_{2}$ agree on a domain on which $T_{2}$ is essentially self-adjoint. As this domain is spanned algebraically by the $x_{n(\cdot)}$ defined previously, it suffices to show that $D Q(x) x_{n(\cdot)}=\left(f_{x} /(2 c)^{(1 / 2)}\right) D x_{n(\cdot)}$. It is readily computed now that $2^{(1 / 2)} i Q(x) x_{n(\cdot)}=(n(\nu)+1)^{(1 / 2)} x_{n^{\prime}(\cdot)}-(n(\nu))^{(1 / 2)} x_{n^{\prime \prime}}(\cdot)$, where $n^{\prime}(\mu)=n(\mu)+\delta_{\mu \nu}$ while $n^{\prime \prime}(\mu)=n(\mu)-\delta_{\mu \nu}$, where the convention is used that if $n(\mu)<0$ for any $\mu$, then $x_{n(\cdot)}=0$.

It follows that $i 2^{(1 / 2)} D Q(x) x_{n(\cdot)}=(n(\nu)+1)^{(1 / 2)} D_{x_{n^{\prime}}(\cdot)}-(n(\nu))^{(1 / 2)} D x_{n^{\prime \prime}(\cdot)}$ $=(n(\nu)+1)^{(1 / 2)} \prod_{\mu} r_{n^{\prime}(\mu)} W_{c / 2}\left(\hat{x}^{n^{\prime}(\mu)}\right)-(n(\nu))^{(1 / 2)} \prod_{\mu} r_{n^{\prime \prime}(\mu)} W_{c / 2}\left(\hat{x}^{n^{\prime \prime}(\mu)}\right)$. On the other hand, $M D x_{n(\cdot)}$ can be expressed correspondingly from the formula $\sum_{n}\left(s^{n} / n !\right) W_{c / 2}\left(\hat{x}^{n}\right)=\exp \left[(1 / 2) c s^{2}+i s \hat{x}\right]$ obtained earlier. Differentiating with respect to $s$ and comparing coefficients yields the equation

$$
\hat{x} W_{c / 2}\left(\hat{x}^{n}\right)=i c n W_{c / 2}\left(\hat{x}^{n-1}\right)-i W_{c / 2}\left(\hat{x}^{n+1}\right)
$$

$(n=0,1,2, \cdots$, a negative index as usual indicates a vanishing quantity). From this it follows by a simple computation that

$$
\hat{x} D x_{n(\cdot)}=-i c^{(1 / 2)}\left[(n(\nu)+1)^{(1 / 2)} D x_{n^{\prime}(\cdot)}-(n(\nu))^{(1 / 2)} D x_{n^{\prime \prime}(\cdot)}\right] .
$$

Thus $2^{(1 / 2)} D Q(x) D^{-1}$ and multiplication by $\hat{x} /(2 c)^{(1 / 2)}$ agree on $\varepsilon$.

Corollary 3.5. The restrictions of $P(x)$ and $Q(x)$ to the algebraic elements are essentially self-adjoint, for all $x$ in $\mathfrak{H}^{\prime}\left({ }^{9}\right)$.

In the case of $Q(x)$ this was shown in the proof of the preceding corollary. As $-P(x)$ is the transform of $Q(x)$ under the Wiener transform (i. e. equals $\left.W_{c} Q(c) W_{c}^{-1}\right)$, and since this operation leaves the set of all algebraic elements invariant, the result for $P(x)$ follows from that for $Q(x)$.

CoROllary 3.6. Let $\left\{x_{\mu}\right\}$ be an orthonormal basis for $\mathcal{H C}^{\prime}$. The symmetric algebra over the complexification of $\mathrm{JC}^{\prime}$ is unitarily equivalent to the space $L_{2}(\Xi)$ over the direct product of $\operatorname{dim} \mathrm{JC}^{\prime}$ copies of the real line with the element of measure $(2 \pi c)^{-(1 / 2)} \exp \left[-x^{2} / 2 c\right] d x$ in such a way that $(2 c)^{1 / 2} Q\left(x_{\mu}\right)$ transforms into multiplication by the $\mu$ th coordinate $\xi_{\mu}$ (where $\xi=\left\{\xi_{\mu}\right\}$ is the generic element of $\Xi$ )

( $\left.{ }^{9}\right)$ Cook [2] was apparently the first to show that the $P(x)$ and $Q(x)$ are self-adjoint. 
and $P\left(x_{\mu}\right)$ goes into an operator that acts on the transforms of polynomials in the functionals $\hat{x}_{\nu}$ as follows:

$$
f \rightarrow-i(2 c)^{(1 / 2)} \frac{\partial f}{\partial \xi_{\mu}}+i(2 c)^{-(1 / 2)} \xi_{\mu} f
$$

(any such $f$ necessarily depending on only a finite number of the $\left.\xi_{\mu}\right)\left({ }^{10}\right)$.

The functions $k_{n(\cdot)}(\xi)=\prod_{\nu} k_{n(\nu)}\left(\xi_{\nu}\right)$ (where the terms in the product for which $n(\nu)$ is zero may be disregarded) form an orthonormal basis in $L_{2}(\Xi)$, where $k_{p}=r_{p} W_{c / 2}\left(x^{p}\right)$, identifying the reals with a one-dimensional real Hilbert space in the usual manner. The proof of Corollary 3.4 shows that the unique unitary map $Y$ of $S$ onto $L_{2}(\Xi)$ that takes $x_{n(\cdot)}$ into $k_{n(\cdot)}$ transforms $Q\left(x_{\mu}\right)$ as stated. Now differentiating the equation

$$
\sum\left(s^{n} / n !\right)\left(W_{c / 2}\left(x^{n}\right)\right)(y)=\exp \left[(1 / 2) c s^{2}+i s y\right]
$$

( $x$ is here a bound variable) with respect to $y$ shows that in the one-dimensional case

$$
\frac{d}{d x} W_{c / 2}\left(u^{n}\right)=i n W_{c / 2}\left(u^{n-1}\right),
$$

$d / d x$ denoting ordinary differentiation. From this it can be inferred as in the proof of Corollary 3.3 that

$-2 i c^{1 / 2} \frac{\partial}{\partial \xi_{\nu}} Y x_{n(\cdot)}+i c^{-(1 / 2)} \xi_{\nu} Y x_{n(\cdot)}=(n(\nu)+1)^{(1 / 2)} Y x_{n^{\prime}(\cdot)}+(n(\nu))^{(1 / 2)} Y x_{n^{\prime \prime}(\cdot) \cdot}$

5. Representations of the additive group of Hilbert space. In the preceding section the action of the duality transform on the symmetric algebra $Q$ 's was fully described, but the action on the $P$ 's was given in a strict way only on a certain subdomain, and in a fashion that depended on a choice of basis. In the present section the action on the $P$ 's is gone into. It is seen to be closely related to the "regular representation" of the additive group of the Hilbert space. Certain aspects of harmonic analysis on this group are also treated.

For any polynomial $f$ over $\mathcal{F}^{\prime}$ and vector $a$ in $\mathcal{F}^{\prime}$ the strict functional $f_{a}$, where $f_{a}(x)=f(x+a)$, is again a polynomial and the map $f \rightarrow f_{a}$ is an algebraic isomorphism, but it is not an isometry. In order to obtain an isometry similar to the familiar isometry induced by vector translation on functions over euclidean space, a factor that plays the part of the square-root of a Radon-Nikodym derivative must be introduced. (In fact, it is precisely this, but as only a special case is involved here there is at present no need to treat

$\left({ }^{10}\right)$ Variants of this result have often been used unrigorously (and in fact incorrectly) in the literature on quantum fields. E.g., $Q\left(x_{n}\right)$ is formally replaced by $\xi_{n}$ and $i P\left(x_{n}\right)$ by $\partial / \partial \xi_{n}$. Actually there is no unitary transformation that eliminates the multiplication part of the $P\left(x_{n}\right)$ and retains the multiplicative aspect of the transforms of the $Q\left(x_{n}\right)$. 
the extension of the Radon-Nikodym theorem to the present type of integration.) The "regular representation" $Y$ of $\mathfrak{F C}^{\prime}$ on $L_{2}\left(\mathcal{F C}^{\prime}, c\right)$ is defined by setting

$$
\left(Y_{0}(a) f\right)(x)=\exp [-(a, x) / 2 c-(a, a) / 4 c] f(x+a)
$$

for $f$ a polynomial, and then extending by continuity to all $f$ in $L_{2}\left(\mathcal{F}^{\prime}, c\right)$. The main properties are stated in

TheOREM 4. $Y$ is a strongly continuous representation of $\mathcal{H C}^{\prime}$ on $L_{2}\left(\mathcal{H C}^{\prime}, c\right)$. The self-adjoint generator of the one-parameter group $Y(t x),-\infty<t<\infty$, is the duality transform of $P(x)(2 c)^{(1 / 2)}$.

First the validity of the foregoing definition of $Y(a)$ is verified. It suffices to show that $Y_{0}(a)$ is isometric on $P$ into $L_{2}\left(\mathcal{F C}^{\prime}, c\right)$, and for this it suffices in turn to show that $Y_{0}(a)$ preserves inner products of monomials relative to arbitrary orthonormal bases of $\mathfrak{F C}^{\prime}$. Let $f$ and $g$ be such monomials, so that $f(x)=\prod_{k=1}^{r}\left(x, x_{k}\right)^{m_{k}}$ and $g(x)=\prod_{k=1}^{r}\left(x, x_{k}\right)^{n_{k}}$, where $x_{1}, \cdots, x_{k}$ are a finite orthonormal set of vectors in $\mathcal{F C}^{\prime}$ and the $m_{k}$ and $n_{k}$ are non-negative integers. Let $a$ be arbitrary in $\mathcal{H}^{\prime}$, and let $x_{r+1}$ be chosen in $\mathfrak{F C}^{\prime}$ so that $a=\sum_{k=1}^{r+1} t_{k} x_{k}$ for suitable real $t_{k}$, while the $x_{1}, x_{2}, \cdots, x_{r+1}$ constitute an orthonormal set-it is assumed here that $\operatorname{dim} \mathfrak{H C}^{\prime}>r$, the modifications required for treating the exceptional case being easily made. Then $Y_{0}(a) f$ and $Y_{0}(a) g$ are based on the finite-dimensional manifold spanned by $x_{1}, \cdots, x_{r+1}$, and their inner product can be expressed as a Lebesgue integral over the corresponding euclidean space: $\left(Y_{0}(a) f, Y_{0}(a) g\right)$ $=\int \prod_{k=1}^{r+1}\left(t_{k}+s_{k}\right)^{m_{k}+n}{ }_{k} \exp \left[-t_{k} s_{k} / c-t_{k}^{2} / 2 c-s_{k}^{2} / 2 c\right](2 \pi c)_{-}{ }^{(1 / 2)} d s_{k}$, which the transformation $s_{k} \rightarrow s_{k}+t_{k}$ shows is independent of the value of $a$. As $Y_{0}(0)$ is obviously the identity on $\mathcal{P}$, this means that $Y_{0}(a)$ preserves the relevant inner products. Thus $Y(a)$ is well-defined and isometric on $L_{2}\left(\mathfrak{H}^{\prime}, c\right)$ into itself.

Now there is no difficulty in showing that if $f$ is a tame functional in $L_{2}\left(H^{\prime}\right)$ (dropping the parameter $c$, which will be constant in the remainder of the paper), then the action of $Y(a)$ on $f$ can be described in the same pointwise terms as those describing the action of $Y_{0}(a)$ on $P$. Moreover, for any vector $b$ in $\mathcal{F C}^{\prime}, Y(b) f$ likewise is tame, so that $Y(a) Y(b) f$ can be computed in pointwise terms. It can thereby be verified that $Y(a) Y(b) f=Y(a+b) f$, which implies that $Y(a+b)=Y(a) Y(b)$. In particular, $Y(a) Y(-a)$ $=Y(-a) Y(a)=I$, which shows that the $Y(a)$ are invertible, and hence unitary.

Thus $Y$ is a unitary representation of the additive group of $\mathcal{F C}^{\prime}$ on $L_{2}\left(\mathcal{H C}^{\prime}, c\right)$. The assertion that $Y$ is strongly continuous means that for every functional $f$ in $L_{2}\left(\mathcal{F C}^{\prime}\right)$, the map $a \rightarrow Y(a) f$ from $\mathfrak{F}^{\prime}$ into $L_{2}\left(\mathfrak{F C}^{\prime}\right)$ is continuous. Since the $Y(a) f$ with arbitrary $f$ in $L_{2}(\mathcal{F})$ are uniform limits of the $Y(a) f$ with $f$ in $\mathcal{P}$, it suffices to check the continuity of the foregoing map for the case when $f$ is in $P$. Moreover, because $Y$ is a representation, continuity at any one point 
implies continuity everywhere. Hence to prove the strong continuity of $Y$ it need only be shown that $Y(a) f$ is a continuous function of $a$ at the point $a=0$, for a fixed functional $f$ that is a monomial relative to some orthonormal base. Now as $Y(a)$ is unitary, $|Y(a) f-f|^{2}=2|f|^{2}-2 \operatorname{Re}[(Y(a) f, f)]$, so it suffices to show that $(Y(a) f, f) \rightarrow(f, f)$ as $a \rightarrow 0$. Let $f$ have the form used in the proof that $Y_{0}(a)$ is isometric, and let $x_{r+1}$, the $s_{k}$, and the $t_{k}$ be as in that proof. Then

$$
(Y(a) f, f)=\int \prod_{k=1}^{r+1}(2 \pi c)-^{(1 / 2)}\left(s_{k}+t_{k}\right)^{m_{k}} \exp \left[-s_{k} t_{k} /(2 c)-t_{k}^{2} /(4 c)-s_{k}^{2} /(2 c)\right] d s_{k}
$$

which is easily seen to have the form $p\left(t_{1}, \cdots, t_{r}\right) \exp \left[-|a|^{2} / 8 c\right]$, where $p\left(t_{1}, \cdots, t_{r}\right)$ is a polynomial in the $t_{k}$. It follows that $(Y(a) f, f)$ is a continuous function of $a$.

It remains only to show that the generator $A$ of the one-parameter group $Y(t x),-\infty<t<\infty$, is the same as the duality transform $B=D P(x) D^{-1}$ of $P(x)$. As both $A$ and $B$ are self-adjoint, it suffices to show that $A$ extends the restriction $B_{1}$ of $B$ to a domain on which $B$ is essentially self-adjoint-for taking closures leads from the inclusion $B_{1} \subset A$ to the inclusion $B \subset A$, which implies that $A=B$. Now by Lemma 3.4.1, $D Q(x) D^{-1}$ is essentially self-adjoint on the domain $\varepsilon$ that occurs in the proof of Corollary 3.4. Now the Wiener transform of $\varepsilon$ is again $\varepsilon$, and it follows that $D P(x) D^{-1}$ is also essentially self-adjoint on $\varepsilon$, using Corollary 3.3. Now since the domain of $A$ consists of all functionals $f$ such that $\lim _{t \rightarrow 0} t^{-1}\left[e^{i A t}-I\right] f$ exists, it follows that it suffices to show the following: if $f=D x_{n(\cdot)}$, then $\lim _{t \rightarrow 0} t^{-1}[Y(t x)-I] f$ exists and equals $D P(x) D^{-1} f$.

For any element $g$ of $L_{2}\left(\mathfrak{F}^{\prime}\right)$ based on the finite-dimensional manifold spanned by $x_{1}, \cdots, x_{r}$, taking among these $x$ as well as the $x_{\mu}$ such that $n(\mu)>0$, let $\tilde{g}$ denote the function on $r$-dimensional euclidean space given by the equation $\tilde{g}\left(t_{1}, \cdots, t_{r}\right)=g\left(t_{1} x_{1}+\cdots+t_{r} x_{r}\right)$. Then the norm of $g$ is the same as the norm of $\tilde{g}$ in the space of square-integrable functions on euclidean $r$-space relative to the volume element $\prod_{k}(2 \pi c)^{-(1 / 2)} e^{-t^{2} k /(2 c)} d t_{k}$. Applying this fact to the functional $g=(i t)^{-1}[Y(t x)-I] f$, which is based on the submanifold in question, shows that convergence in mean of $h_{t}(s)$ as $t \rightarrow 0$, where $h_{t}(s)=(i t)^{-1}\left[\tilde{g}\left(s_{1}+t, s_{2}, \cdots, s_{r}\right) \exp \left[s_{1} t /(2 c)-t^{2} /(4 c)\right]-g\left(s_{1}, s_{2}, \cdots, s_{r}\right)\right]$ is equivalent to the convergence of $(i t)^{-1}[Y(t x)-I] f$. As $\tilde{g}$ is continuously differentiable, $h_{t}$ converges pointwise as $t \rightarrow 0$ to

$$
\frac{\partial}{\partial t}\left[\tilde{g}\left(s_{1}-t, s_{2}, \cdots, s_{r}\right) \exp \left(s_{1} t /(2 c)-t^{2} /(4 c)\right)\right]_{\ell=0} .
$$

On the other hand, $h_{t}$ is uniformly dominated, for $|t|<1$, by the squareintegrable function

$$
\sup _{|\imath| \leqq 1} \mid \tilde{g}\left(s_{1}-t, s_{2}, \cdots, s_{r}\right)\left(\exp \left[\left|s_{1}\right| /(2 c)\right]+1\right) .
$$


It follows that $h_{t}$ converges in mean to $-i\left[\left(\partial / \partial s_{1}\right) \tilde{g}-\left(s_{1} /(2 c)\right) g\right]$. From Corollary 3.6 it is clear that this function has the form $k$, where $k=c^{-(1 / 2)} D P(x) D^{-1} f$ which implies that $(i t)^{-1}[Y(t x)-I] f \rightarrow(2 c)^{-(1 / 2)} D P(x) D^{-1} f$ as $t \rightarrow 0$.

Corollary 4.1. For any $x$ and $y$ in $\mathfrak{F}^{\prime}$,

$\exp (-i P(x)) \exp (i Q(y)) \exp (i P(x))=\exp [-(x, y)] \exp (i Q(y))\left({ }^{11}\right)$.

The verification of this corollary involves a straightforward computation based on the preceding theorem and the fact (implied by Corollary 3.4) that $\exp (i Q(y))$ is the result of applying the duality transform to the operation of multiplication by $\exp \left(i f_{y} /(2 c)^{(1 / 2)}\right.$.

At this point it would be possible to develop a variety of features of harmonic analysis over a Hilbert space, including extension of properties of convolutions, but the only additional result that will be presented here is an extension to the theorem of Stone giving the structure of arbitrary unitary representations of the additive group of the reals, or of a finite-dimensional real vector space; this extension is pertinent to a technical question concerning representations of systems similar to a symmetric algebra, which will be treated elsewhere. In Stone's theorem a representation is determined by a projection-valued measure on the group. The situation is essentially the same here, except that a type of weak measure is used satisfying a certain continuity restriction.

Definition 4. A "weak resolution" over $\mathfrak{H C}^{\prime}$ of the identity on the Hilbert space $\mathcal{L}$ is a linear map from $\mathfrak{H}^{\prime}$ to an abelian (measurable) family of selfadjoint operators on $\mathcal{L}$.

The term "measurable" is used in the sense of [8], whose notions can however be avoided here as in the present situation abelian rings only are involved. Two self-adjoint operators are here said to commute if any two of their spectral projections commute, and an abelian family is one such that any two elements of it commute. A maximal abelian set of self-adjoint operators constitutes a real linear associative algebra with respect to "strong" operations, in which the customary operation for operators is followed by that of taking the closure. It is with respect to these strong operations that the linearity of the map in Definition 4 is taken. In other terms, if $x \rightarrow A$ and $y \rightarrow B$, then $x+y \rightarrow$ closure of $A+B$ and $t x \rightarrow$ closure of $t A$ for all real $t$ (including $t=0$ ).

Weak resolutions and weak distributions over $\mathcal{F C}^{\prime}$ are closely related, in fact a common generalization could readily be treated, by assuming the existence of a distinguished trace (either numerical- or operator-valued) on the relevant abelian ring of operators, but for present purposes at least it seems simpler to treat the two notions separately.

(11) These are the field commutation relations in the bounded form of Weyl. Apparently no rigorous proof that they hold in a boson field has previously been given. 
The relation between weak resolutions and projection-valued measures is shown by the fact that if $F$ is a weak solution over $\mathcal{H C}^{\prime}$ of the identity on $\mathcal{L}$, there exists a unique related map $\widetilde{F}$ from the tame functions on $\mathcal{F}^{\prime}$ to the normal operators in an abelian family containing the range of $F$, such that for any Baire function $\phi$ on euclidean $n$-space and vectors $x_{1}, \cdots, x_{n}$ in $\mathfrak{H C}^{\prime}$,

$$
\phi\left(F\left(x_{1}\right), \cdots, F\left(x_{n}\right)\right)=\tilde{F}\left(\phi\left(\hat{x}_{1}, \cdots, \hat{x}_{n}\right)\right),
$$

where $\hat{x}_{k}$ is the functional defined by the equation $\hat{x}_{k}(u)=\left(u, x_{k}\right)$; and $\tilde{F}$ necessarily takes characteristic functions into projections. By virtue of the last property, $F$ induces in a canonical way an additive map $F$ from the tame subsets of $\mathfrak{H C}^{\prime}$ to the projections on $\mathcal{L}$ that is countably additive on the tame sets based on a fixed finite-dimensional manifold and takes the entire space into the identity operator. Conversely, a projection-valued measure on the tame subsets of $\mathcal{F C}^{\prime}$ with the properties just mentioned necessarily arises from a unique weak resolution in the fashion described. These results follow in a standard way from well-known features of the spectral resolution of abelian rings of operators, so that details of the proof are omitted.

It is suggestive to write $\widetilde{F}(f)$ in the form $\int f(u) d \breve{F}(u), \breve{F}$ being defined as indicated above, i. e. it takes a tame subset $A$ into $\widetilde{F}\left(c_{A}\right)$, where $c_{A}$ denotes the characteristic function of $A$.

The resolutions and distributions that are relevant to the extension of Stone's theorem are not arbitrary but must satisfy a continuity condition, which is trivially fulfilled in the case of a finite-dimensional group.

Definition 5. A weak distribution or resolution $F$ over $\mathcal{H}^{\prime}$ is said to be "continuous" if whenever $x_{n} \rightarrow x$ in $\mathcal{F C}^{\prime}, F\left(x_{n}\right) \rightarrow F(x)$ in measure or asymptotically respectively-where a sequence $\left\{A_{n}\right\}$ of commutative self-adjoint operators is said to converge asymptotically to a self-adjoint operator $A$ commuting with all of them if for every positive $\epsilon$, the projections $P_{n}(\epsilon)$ tend weakly to zero as $n \rightarrow \infty$, where $P_{n}(\boldsymbol{\epsilon})=\int_{|t|>\epsilon} d E_{n}(t)$ and the spectral resolution of the strong difference $A_{n}-A$ is $\int t d E_{n}(t)$.

THEOREM 5. For any strongly continuous unitary representation $Y$ of the additive group of the real Hilbert space $\mathfrak{H C}^{\prime}$ there exists a unique continuous weak resolution $F$ over $\mathfrak{H}^{\prime}$ such that $Y(x)=\int \mathcal{H C} \cdot e^{i(x, y)} d F(y)$. Conversely, if $F$ is a continuous weak resolution over $\mathcal{H}^{\prime}$, the foregoing equation defines a strongly continuous unitary representation of ' $\mathfrak{H C}^{\prime}$.

The direct part of the theorem may be proved by first showing that any continuous normalized positive definite function on $\mathcal{F C}^{\prime}$ is the Fourier-Stieltjes transform of a weak distribution (in the straightforwardly generalized sense), as in a well-known approach to Stone's theorem. It is shorter to derive it directly from Stone's theorem [10].

Let $Y$ be a strongly continuous unitary representation of $\mathfrak{H C}^{\prime}$ on the 
Hilbert space $\mathcal{L}$, and let $\mathcal{X}^{\prime}$ be an arbitrary finite-dimensional subspace of $\mathfrak{H}^{\prime}$. By Stone's theorem there exists a weak resolution $F \mathcal{X}$, over $\mathcal{H}^{\prime}$ of the identity on $\mathcal{L}$ such that for $x$ in $\mathcal{X}^{\prime}$,

$$
Y(x)=\int_{\mathcal{H}^{\prime}} e^{i(x, y)} d \breve{F}_{\mathcal{X}}(y) .
$$

It follows from the uniqueness of $F \mathcal{F} C$, that if $\mathcal{N}^{\prime}$ is a finite-dimensional subspace containing $\mathcal{X}^{\prime}$, then $F \mathcal{N}^{\prime}$ is an extension of $F \mathcal{X} \mathcal{C}^{\prime}$. Putting $F$ for the common extension to all of $\mathcal{F C}^{\prime}$ of all of the $F \mathcal{F} \mathcal{C}^{\prime}$, it results that $Y(x)$ has the stated form. It follows also from Stone's theorem that the operators in the range of $F$ have their spectral projections in the ring generated by the $Y(x)$.

It remains to show that $F$ is continuous, for which it suffices to show that if $x_{n} \rightarrow 0$, then $F\left(x_{n}\right) \rightarrow 0$ asymptotically. By polarization, this is equivalent to the statement that $\left(P_{n}(\epsilon) a, a\right) \rightarrow 0$ for each vector $a$ in the representation space, where $P_{n}(\epsilon)$ is as defined above. Now note that there exists for any $\epsilon>0$ a $p>0$ such that

$$
(P a, a) \leqq p \int_{-\epsilon}^{\epsilon}\left(\left(I-e^{i t A}\right) a, a\right) d t
$$

for an arbitrary self-adjoint operator $A$, where $P=c_{\epsilon}(A), c_{\epsilon}$ denoting the characteristic function of the complement of $(-\epsilon, e)$. For the spectral theorem shows that this inequality follows from the simple inequality $c_{\epsilon}(s)$ $\leqq p \int_{-\epsilon}^{e}\left(1-e^{i t s}\right) d t$. In particular,

$$
\left(P_{n}(\epsilon) a, a\right) \leqq p \int_{-\epsilon}^{\epsilon}\left(\left(I-\exp \left(i t F\left(x_{n}\right)\right)\right) a, a\right) d t,
$$

and as it results from the definition of $\widetilde{F}$ that $e^{i t F(x)}=Y(x)$, it is easy to conclude from the weak continuity of $Y$ and the bounded convergence theorem that $\left(P_{n}(\epsilon) a, a\right) \rightarrow 0$ as $n \rightarrow \infty$.

The only part of the converse that is not immediate from well-known features of spectral resolutions is the strong continuity. As the weak and strong operator topologies coincide on the unitary group it suffices to show weak continuity, and by polarization, etc., it suffices to show that for every unit vector $a$ in $\mathfrak{H C}^{\prime}$ the representation space, the map $x \rightarrow(Y(x) a, a)$ is continuous. But it is straightforward that $(Y(x) a, a)=\int e^{i(x, y)} d F_{a}(y)$, where $F_{a}$ is the weak distribution on $\mathfrak{H}^{\prime}$ whose mapping is $F$, and where the bounded operators in the ring generated by the bounded Baire functions of the $F(x)$ are formulated as a probability algebra by assigning the expectation $(T a, a)$ to any such operator $T$. Now the continuity of the weak resolution $F$ directly implies that of the weak distribution $F_{a}$, for all $a$, and hence it suffices to show that if $G$ is a continuous weak distribution on $\mathcal{H}^{\prime}$, then the integral relative to $G$ of $e^{i x}$, where $\hat{x}$ denotes the functional defined by the equation $\hat{x}(y)=(y, x)$, 
depends continuously on $x$. This follows directly from the fact that if $\left\{f_{n}\right\}$ is any bounded sequence of real-valued random variables on a probability space converging in measure to a function $f$, then $\int f_{n} \rightarrow \int f$.

\section{REFERENCES}

1. R. H. Cameron and W. T. Martin, Fourier-Wiener transforms of functionals belonging to $L_{2}$ over the space $C$, Duke Math. J. vol. 14 (1947) pp. 99-107.

2. J. M. Cook, The mathematics of second quantization, Trans. Amer. Math. Soc. vol. 74 (1953) pp. 222-245.

3. P. A. M. Dirac, The principles of quantum mechanics, 3d ed., Oxford, 1947.

4. R. P. Feynman, An operator calculus having applications in quantum electrodynamics, The Physical Review vol. 84 (1951) pp. 108-128.

5. K. O. Friedrichs, Mathematical aspects of the quantum theory of fields, New York, 1953.

6. S. Kakutani, Determination of the spectrum of the flow of Brownian motion, Proc. Nat. Acad. Sci. vol. 36 (1950) pp. 319-323.

7. F. J. Murray and J. von Neumann, On rings of operators, Ann. of Math. vol. 37 (1936) pp. 116-229.

8. I. E. Segal, A non-commutative extension of abstract integration, Ann. of Math. vol. 57 (1953) pp. 401-457.

9. - - Abstract probability spaces and a theorem of Kolmogoroff, Amer. J. Math. vol. 76 (1954) pp. 721-732.

10. M. H. Stone, On one-parameter unitary groups in Hilbert space, Ann. of Math. vol. 33 (1932) pp. 643-648.

11. H. Weyl, The classical groups, Princeton, 1939.

12. - Gruppentheorie und Quantenmechanik, 2d ed., Leipzig, 1931.

13. N. Wiener, The homogeneous chaos, Amer. J. Math. vol. 60 (1939) pp. 897-936.

Columbia University,

NEW YORK, N. Y.

Institute for Advanced Study,

Princeton, N. J.

University OF Chicago,

Chicago, Ill. 